\title{
Basic Operation of Cryocoolers and Related Thermal Machines
}

\author{
A.T.A.M. de Waele
}

Received: 3 March 2011 / Accepted: 15 May 2011 / Published online: 10 June 2011

(C) The Author(s) 2011. This article is published with open access at Springerlink.com

\begin{abstract}
This paper deals with the basics of cryocoolers and related thermodynamic systems. The treatment is based on the first and second law of thermodynamics for inhomogeneous, open systems using enthalpy flow, entropy flow, and entropy production. Various types of machines, which use an oscillating gas flow, are discussed such as: Stirling refrigerators, GM coolers, pulse-tube refrigerators, and thermoacoustic coolers and engines. Furthermore the paper deals with Joule-Thomson and dilution refrigerators which use a constant flow of the working medium.
\end{abstract}

Keywords Thermodynamics · Cryocoolers · Thermoacoustics · Dilution refrigerators

\section{Introduction}

This paper deals with the basics of cryocoolers and related thermodynamic systems. A cryocooler is a standalone cooler of table-top size which is used to cool some particular application. Reference [1] is a recent review. In this paper the essence of the system operation will be discussed. Whenever possible mathematical complications will be avoided. Distracting practical effects, such as high-order disturbances, streaming, heat leaks, transient effects, etc. will be neglected. The starting point will be the first and second law of thermodynamics for inhomogeneous, open systems [2]. Important concepts as enthalpy flow, entropy flow, and entropy production will be introduced. Thoroughly understanding thermal machines (coolers and heat engines) without these concepts is impossible.

After the introduction and a short discussion of ideal regenerators and heat exchangers, various types of coolers are discussed which operate with oscillating flows.

A.T.A.M. de Waele ( $₫)$

Eindhoven University of Technology, PO Box 513, 5600MB Eindhoven, The Netherlands

e-mail: a.t.a.m.d.waele@tue.nl 


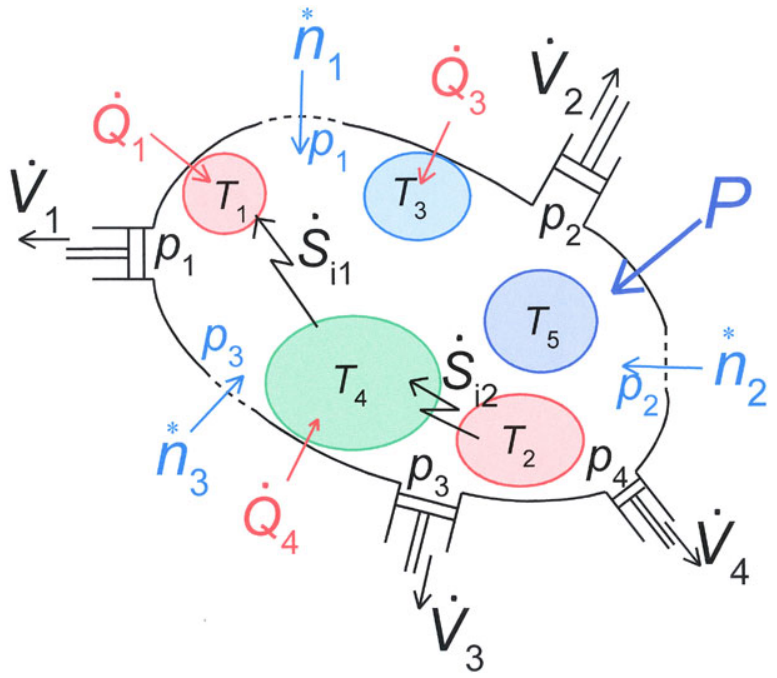

Fig. 1 (Color online) General representation of a system that consists of a number of subsystems. The interaction with the surroundings of the system can be in the form of exchange of heat and other forms of energy, exchange of matter, and change of shape. The interactions between the subsystems are of a similar nature and lead to entropy production. In this figure the $\dot{V}_{\mathrm{k}}$ stand for $d V_{\mathrm{k}} / d t$

Next, properties are discussed of less ideal regenerators, followed by a treatment of thermoacoustic coolers and engines. The paper ends with the discussion of JouleThomson coolers and dilution refrigerators which operate with a steady flow of the working fluid. Appendix A gives some useful thermodynamic formulae, Appendix B is a derivation of the so-called volume-flow equation, and Appendix $\mathrm{C}$ is about the harmonic model.

\section{The First and Second Law of Thermodynamics}

The laws of thermodynamics apply to well-defined systems. Figure 1 is a general representation of a thermodynamic system. We consider systems which can be inhomogeneous. We allow heat and mass transfer across the boundary (nonadiabatic, open systems), and we allow the boundary to move. In our formulation we will assume that heat and mass transfer and volume changes take place only at some well-defined regions of the system boundary. Equations (1) and (4) are not the most general formulations of the first and second law. E.g. kinetic energy terms are missing and exchange of matter by diffusion is excluded.

\subsection{First Law}

The first law reads

$$
\frac{\mathrm{d} U}{\mathrm{~d} t}=\sum_{k} \dot{Q}_{k}+\sum_{k} \stackrel{*}{H}_{k}-\sum_{k} p_{k} \frac{\mathrm{d} V_{k}}{\mathrm{~d} t}+P .
$$


In (1) $t$ is time,

- $U$ is a function of state, called the internal energy of the system.

- $\dot{Q}_{k}$ are the heat flows into the system at the various regions of the boundary which are labeled with $k$.

- $\stackrel{*}{H}_{k}$ are the enthalpy flows into the system due to the matter that flows into the system. It is defined as

$$
\stackrel{*}{H}_{k}=\stackrel{*}{n}_{k} H_{\mathrm{m} k}=\stackrel{*}{m} h_{k},
$$

where $\stackrel{*}{n}_{k}$ is the molar flow of matter flowing into the system and $H_{\mathrm{m} k}$ its molar enthalpy, ${ }^{1} h_{k}$ the specific enthalpy (i.e. enthalpy per unit mass), and

$$
\stackrel{*}{m}_{k}=M_{k} \stackrel{*}{n}_{k}
$$

the mass flow with $M_{k}$ the molar mass.

- $\mathrm{d} V_{k} / \mathrm{d} t$ are the rates of change of the volume of the system due to the various moving boundaries, $p_{k}$ is the pressure behind boundary $k$.

- $P$ takes into account all other forms of power applied to the system by its environment (such as electrical, shaft power, etc.).

We use the notation $\stackrel{*}{Y}$ for the flow of a thermodynamic state function $Y$ and $\mathrm{d} Y / \mathrm{d} t$ for the rate of change of $Y$. Even though the dimensions of ${ }^{*}$ and $\mathrm{d} Y / \mathrm{d} t$ are the same their physical meaning is distinctly different. For the heat flow we use the dot notation.

\subsection{Second Law}

The second law reads

$$
\frac{\mathrm{d} S}{\mathrm{~d} t}=\sum_{k} \frac{\dot{Q}_{k}}{T_{k}}+\sum_{k} \stackrel{*}{S}_{k}+\sum_{k} \dot{S}_{\mathrm{i} k} \quad \text { with } \dot{S}_{\mathrm{i} k} \geq 0 .
$$

Here

- $S$ is a function of state, called the entropy of the system.

- $T_{k}$ represent the temperatures at which the heat flows $\dot{Q}_{k}$ enter the system.

- $\stackrel{*}{S}_{k}$ represent the entropy flows into the system, due to matter flowing into the system, given by

$$
\stackrel{*}{S_{k}}=\stackrel{*}{n_{k}} S_{\mathrm{m} k}=\stackrel{*}{m_{k}} s_{k} .
$$

Here $S_{\mathrm{m} k}$ and $s_{k}$ are the molar and specific entropy of the matter, flowing into the system, respectively.

\footnotetext{
${ }^{1}$ We will use the lower index $\mathrm{m}$ to indicate molar quantities. In many thermoacoustic papers the lower index $\mathrm{m}$ is used to indicate the mean value. For the latter we will use the lower index 0 .
} 


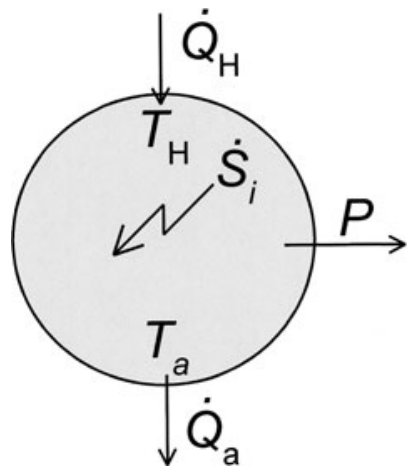

Fig. 2 Schematic diagram of a heat engine. A heating power $\dot{Q}_{\mathrm{H}}$ enters the system at some high temperature $T_{\mathrm{H}}$, and $\dot{Q}_{\mathrm{a}}$ is released at ambient temperature $T_{\mathrm{a}}$. A power $P$ is produced and the entropy production rate is $\dot{S}_{\mathrm{i}}$

- $\dot{S}_{\mathrm{i} k}$ represent the entropy production rates due to internal processes. Each of the entropy production rates is always positive or zero. This is an essential aspect of the second law. The summation is over all processes in the system. The most important irreversible processes, for our application, are heat flow over a temperature difference and mass flow over a pressure difference.

In many cases $\dot{Q} / T$ is also considered as an entropy flow which is associated with the heat flow. In this case the second law is a conservation law with flow and source terms.

\subsection{Consequences of the First and Second Law}

\subsubsection{Heat Engines}

Figure 2 is a schematic diagram of a heat engine. The machine is cyclic and in the steady state. A heating power $\dot{Q}_{\mathrm{H}}$ enters the engine at a temperature $T_{\mathrm{H}}$ and a heat flow $\dot{Q}_{\mathrm{a}}$ leaves it at ambient temperature $T_{\mathrm{a}}$. A power $P$ is produced. The sign conventions of $P$ and the heat flows are chosen in such a way that their values are positive under normal system operation. They differ from the signs defined in Fig. 1.

Due to the irreversible processes inside the engine entropy is produced at a rate $\dot{S}_{\mathrm{i}}$. After one cycle, the state of the engine is the same as at the beginning of the cycle, thus, on average, $\mathrm{d} U / \mathrm{d} t=0$ and $\mathrm{d} S / \mathrm{d} t=0$. The system is closed so $\stackrel{*}{H}=0$ and $\stackrel{*}{S}=0$. The boundaries of the system are fixed so $\mathrm{d} V_{k} / \mathrm{d} t=0$. As a result the first law for the engine reduces to

$$
\dot{Q}_{\mathrm{H}}-\dot{Q}_{\mathrm{a}}=P
$$

and the second law to

$$
0=\frac{\dot{Q}_{\mathrm{H}}}{T_{\mathrm{H}}}-\frac{\dot{Q}_{\mathrm{a}}}{T_{\mathrm{a}}}+\dot{S}_{\mathrm{i}} \quad \text { with } \dot{S}_{\mathrm{i}} \geq 0
$$


or

$$
\dot{S}_{\mathrm{i}}=\frac{\dot{Q}_{\mathrm{a}}}{T_{\mathrm{a}}}-\frac{\dot{Q}_{\mathrm{H}}}{T_{\mathrm{H}}} \geq 0 .
$$

If $\dot{Q}_{\mathrm{a}}$ would be zero condition (8) would reduce to

$$
\dot{S}_{\mathrm{i}}=-\frac{\dot{Q}_{\mathrm{H}}}{T_{\mathrm{H}}} \geq 0 .
$$

As $T_{\mathrm{H}}>0$ and $\dot{Q}_{\mathrm{H}} \geq 0$ condition (9) would not be satisfied. So $\dot{Q}_{\mathrm{a}}$ cannot be zero. This means that a heat engine can operate only if heat is released at a low temperature. This is the well-known Kelvin formulation of the second law.

Eliminating $\dot{Q}_{\mathrm{a}}$ from (7), with (6), gives

$$
P=\left(1-\frac{T_{\mathrm{a}}}{T_{\mathrm{H}}}\right) \dot{Q}_{\mathrm{H}}-T_{\mathrm{a}} \dot{S}_{\mathrm{i}} .
$$

As $\dot{S}_{\mathrm{i}} \geq 0$ we must require

$$
P \leq\left(1-\frac{T_{\mathrm{a}}}{T_{\mathrm{H}}}\right) \dot{Q}_{\mathrm{H}} .
$$

The efficiency of a heat engine is defined as

$$
\eta=\frac{P}{\dot{Q}_{\mathrm{H}}} .
$$

With (11) it follows

$$
\eta \leq 1-\frac{T_{\mathrm{a}}}{T_{\mathrm{H}}} .
$$

This famous relation shows that the efficiency of all thermal engines has a maximum given by the Carnot efficiency defined as

$$
\eta_{\mathrm{C}}=1-\frac{T_{\mathrm{a}}}{T_{\mathrm{H}}}
$$

From (10) it can be seen that the efficiency of all heat engines is reduced due to the term $T_{\mathrm{a}} \dot{S}_{\mathrm{i}}$. This important quantity is called the dissipated energy.

\subsubsection{Refrigerators}

Refrigerators, as depicted in Fig. 3, can be treated in a similar way as heat engines. The first law gives

$$
\dot{Q}_{\mathrm{a}}=P+\dot{Q}_{\mathrm{L}}
$$

and the second law

$$
0=\frac{\dot{Q}_{\mathrm{L}}}{T_{\mathrm{L}}}-\frac{\dot{Q}_{\mathrm{a}}}{T_{\mathrm{a}}}+\dot{S}_{\mathrm{i}} \quad \text { with } \dot{S}_{\mathrm{i}} \geq 0
$$




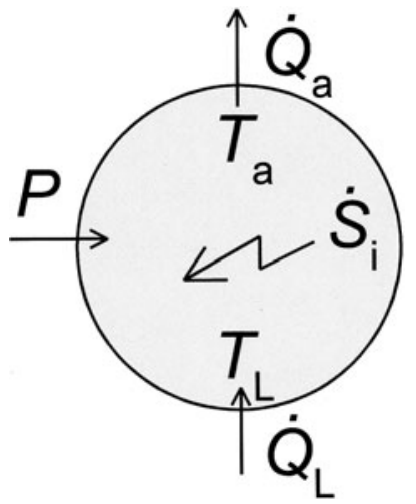

Fig. 3 Schematic diagram of a refrigerator. $\dot{Q}_{\mathrm{L}}$ is the cooling power at some low temperature $T_{\mathrm{L}}$, and $\dot{Q}_{\mathrm{a}}$ is released at ambient temperature $T_{\mathrm{a}}$. A power $P$ is supplied to the system and $\dot{S}_{\mathrm{i}}$ is the entropy production rate

or

$$
\dot{S}_{\mathrm{i}}=\frac{\dot{Q}_{\mathrm{a}}}{T_{\mathrm{a}}}-\frac{\dot{Q}_{\mathrm{L}}}{T_{\mathrm{L}}} \geq 0 .
$$

Eliminating $\dot{Q}_{\mathrm{a}}$ from (17) by (15) gives

$$
\dot{S}_{\mathrm{i}}=\frac{P+\dot{Q}_{\mathrm{L}}}{T_{\mathrm{a}}}-\frac{\dot{Q}_{\mathrm{L}}}{T_{\mathrm{L}}} \geq 0 .
$$

If $P$ would be zero then (18) would reduce to

$$
\dot{S}_{\mathrm{i}}=\left(\frac{1}{T_{\mathrm{a}}}-\frac{1}{T_{\mathrm{L}}}\right) \dot{Q}_{\mathrm{L}} \geq 0 .
$$

This condition is not satisfied since both $\dot{Q}_{\mathrm{L}} \geq 0$ and $T_{\mathrm{a}}>T_{\mathrm{L}}$. This means that heat can not flow from a low temperature to a high temperature without doing work. This is Clausius formulation of the second law.

Turning back to (18), we see that

$$
P=\frac{T_{\mathrm{a}}-T_{\mathrm{L}}}{T_{\mathrm{L}}} \dot{Q}_{\mathrm{L}}+T_{\mathrm{a}} \dot{S}_{\mathrm{i}}
$$

As $\dot{S}_{\mathrm{i}} \geq 0$ we must require

$$
P \geq \frac{T_{\mathrm{a}}-T_{\mathrm{L}}}{T_{\mathrm{L}}} \dot{Q}_{\mathrm{L}}
$$

The Coefficient Of Performance (COP) $\xi$ of coolers is defined as

$$
\xi=\frac{\dot{Q}_{\mathrm{L}}}{P}
$$


With (21) follows

$$
\xi \leq \frac{T_{\mathrm{L}}}{T_{\mathrm{a}}-T_{\mathrm{L}}} .
$$

This relation shows that the $C O P$ of all refrigerators has a maximum given by

$$
\xi_{\mathrm{C}}=\frac{T_{\mathrm{L}}}{T_{\mathrm{a}}-T_{\mathrm{L}}} .
$$

This quantity is called the Carnot $C O P$ of refrigerators. From (20) it can be seen that the $C O P$ of the cooler is reduced by the dissipated energy $T_{\mathrm{a}} \dot{S}_{\mathrm{i}}$.

\subsubsection{The Volume-Flow Equation}

From the first law a very useful expression can be derived which we will call the volume-flow equation. In case of a periodic pressure change, with angular frequency $\omega$, the characteristic length scale for temperature variations of the working fluid is given by the thermal penetration depth $\delta_{\kappa}$ given by

$$
\delta_{\kappa}^{2}=\frac{2 \kappa V_{\mathrm{m}}}{\omega C_{\mathrm{p}}} .
$$

Here $\kappa$ is the thermal conductivity, $V_{\mathrm{m}}$ the molar volume, and $C_{\mathrm{p}}$ the molar heat capacity at constant pressure. Viscous effect are characterized by the viscous penetration depth $\delta_{v}$ given by

$$
\delta_{v}^{2}=\frac{2 \eta}{\omega \rho}
$$

with $\eta$ the viscosity and $\rho$ the density. The two penetration depths are related by the Prandtl number

$$
P_{\mathrm{r}}=\frac{\eta C_{\mathrm{p}}}{M \kappa}
$$

with $M$ the molar mass, as follows

$$
\delta_{v}^{2}=P_{\mathrm{r}} \delta_{\kappa}^{2}
$$

For many working fluids $P_{\mathrm{r}}$ is practically constant and of order 1 , so the two penetration depths are of the same order of magnitude. For helium gas $P_{\mathrm{r}} \approx 0.66$.

For helium, at typical working conditions of cryocoolers, the thermal penetration depth is in the $0.1-0.5 \mathrm{~mm}$ range. Many spaces in thermal machines, such as the compression and expansion spaces in Stirling coolers, the pulse tube in a pulse-tube refrigerator, etc. are much larger than this, so the bulk of the gas is thermally isolated from the surroundings. In other words, in many cases, the pressure changes in the bulk of the gas are adiabatic. Furthermore the pressure $p$ in these spaces is practically homogeneous. Finally, at not too low temperatures and not too high pressures, the gas can be considered to be an ideal gas. Under these conditions a very useful relation 
Fig. 4 Gas flows with varying pressure: (a) inlet and outlet; (b) inlet and piston; (c) fixed volume with valve with flow conductance $C$

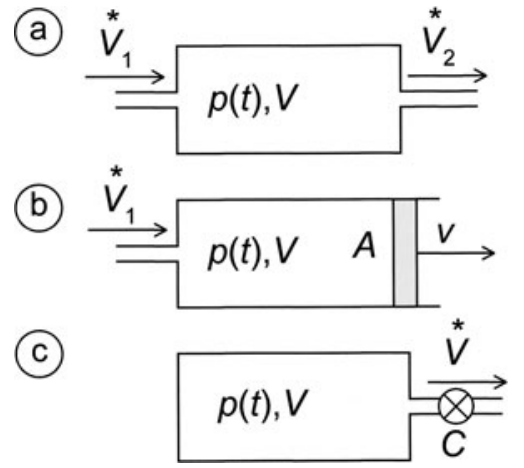

can be derived (Appendix B) which reads as follows

$$
\frac{V}{\gamma p} \frac{\mathrm{d} p}{\mathrm{~d} t}=\sum \stackrel{*}{V}_{k}-\sum \frac{\mathrm{d} V_{k}}{\mathrm{~d} t} .
$$

Here $V$ is the volume of the system, $V_{k}$ are the volume flows at various positions $k$, given by

$$
\stackrel{*}{V}_{k}=\stackrel{*}{n}_{k} V_{\mathrm{m} k},
$$

the $\mathrm{d} V_{k} / \mathrm{d} t$ are the rates of change of the volume due to moving boundaries at position $k$ (usually a piston), and $\mathrm{d} p / \mathrm{d} t$ is the rate of change of the pressure.

If, in addition to the conditions mentioned above, the system is closed all $\stackrel{*}{V}_{k}=0$ so, with (181), Equation (29) reduces to

$$
\frac{V}{\gamma p} \frac{\mathrm{d} p}{\mathrm{~d} t}=-\frac{\mathrm{d} V}{\mathrm{~d} t} \text { (adiabatic, closed). }
$$

From this relation we find

$$
p V^{\gamma}=\text { constant (adiabatic, closed). }
$$

Equation (32) has the same form as the well-known Poisson relation but it has a much wider validity since it includes systems with an inhomogeneous temperature distribution. The Poisson relation is just a special case of (32).

In Fig. 4 three situations are depicted. For the case of Fig. 4a, in which gas flows into and out of a control volume with fixed volume $V$ and with a varying pressure $p(t),(29)$ reads

$$
\stackrel{*}{V}_{1}=\stackrel{*}{V}_{2}+\frac{V}{\gamma p} \frac{\mathrm{d} p}{\mathrm{~d} t} .
$$

This relation holds, in particular, for the pulse tube in a pulse-tube refrigerator (PTR). For the case of Fig. $4 \mathrm{~b}$ gas flows into a control volume with, on the other side, a 
Fig. 5 Heat conduction. The dotted line represents the system. A heat flow $\dot{Q}_{1}$ enters at $T_{1}$ and $\dot{Q}_{2}$ leaves at $T_{2}$

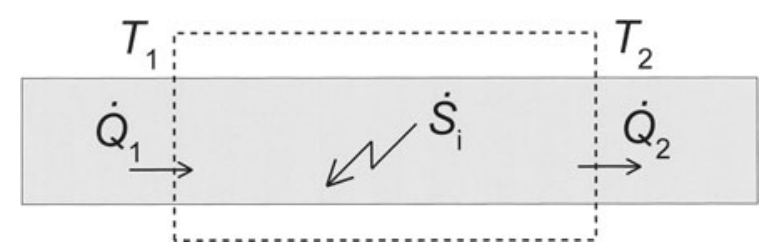

moving piston with area $A$ and velocity $v$. Now (29) reads

$$
\stackrel{*}{V}_{1}=v A+\frac{V}{\gamma p} \frac{\mathrm{d} p}{\mathrm{~d} t} .
$$

For the case of Fig. 4c, where gas flows out of a container through a valve with flow conductance $C$ (as in the buffer volume of a PTR),

$$
0=C\left(p-p_{0}\right)+\frac{V}{\gamma p} \frac{\mathrm{d} p}{\mathrm{~d} t} .
$$

\subsection{Entropy Production Rates}

Now we will derive the expressions for the entropy production rates due to flows of heat and matter.

\subsubsection{Heat Conduction}

Consider the heat conduction through an adiabatic bar in the steady state (see Fig. 5). It is a closed system with fixed boundaries and no external work is done, so the first law reduces to

$$
\dot{Q}_{1}=\dot{Q}_{2}=\dot{Q}
$$

The second law gives

$$
0=\frac{\dot{Q}_{1}}{T_{1}}-\frac{\dot{Q}_{2}}{T_{2}}+\dot{S}_{\mathrm{i}} \quad \text { with } \dot{S}_{\mathrm{i}} \geq 0
$$

or, with (36),

$$
\dot{S}_{\mathrm{i}}=\dot{Q}\left(\frac{1}{T_{2}}-\frac{1}{T_{1}}\right) \geq 0 .
$$

Relation (38) is the expression of the entropy production rate due to transport of heat over a temperature difference. As $\dot{Q}>0$, Equation (38) demands that $T_{1} \geq T_{2}$. In other words: heat flows from a high temperature to a low temperature. This is again the Clausius formulation of the second law.

The heat flow in a bar of length $L$ and cross-sectional area $A$ can, for small temperature differences, be written as

$$
\dot{Q}=\kappa \frac{A}{L}\left(T_{1}-T_{2}\right)
$$


with $\kappa$ the thermal conductivity. The entropy production rate is now

$$
\dot{S}_{\mathrm{i}}=\kappa \frac{A}{L} \frac{\left(T_{1}-T_{2}\right)^{2}}{T_{1} T_{2}} .
$$

The dependence of $\dot{S}_{\mathrm{i}}$ on the "driving force" $\left(T_{1}-T_{2}\right)$ is quadratic, which is characteristic for expressions of the entropy production rates in general [2].

\subsubsection{Throttling}

Now we derive an expression for the entropy production in the throttling process as shown in Fig. 6 . The process is adiabatic by definition. In the steady state

$$
\stackrel{*}{n_{1}}=\stackrel{*}{n_{2}}=\stackrel{*}{n}
$$

The first law demands that

$$
0=\stackrel{*}{n}_{1} H_{\mathrm{m} 1}-\stackrel{*}{n}_{2} H_{\mathrm{m} 2}
$$

so, in a throttling process, the molar enthalpy is constant

$$
H_{\mathrm{m} 1}=H_{\mathrm{m} 2} \text {. }
$$

The second law gives

$$
0=\stackrel{*}{n}_{1} S_{\mathrm{m} 1}-\stackrel{*}{n_{2}} S_{\mathrm{m} 2}+\dot{S}_{\mathrm{i}}
$$

With (43) and (163) we get

$$
\left(\frac{\partial S_{\mathrm{m}}}{\partial p}\right)_{H_{\mathrm{m}}}=-\frac{V_{\mathrm{m}}}{T}
$$

so, with (44),

$$
\dot{S}_{\mathrm{i}}=-\stackrel{*}{n} \int_{1}^{2} \frac{V_{\mathrm{m}}}{T} \mathrm{~d} p .
$$

Introducing the volume flow rate

$$
\stackrel{*}{V}=\stackrel{*}{n} V_{\mathrm{m}}
$$

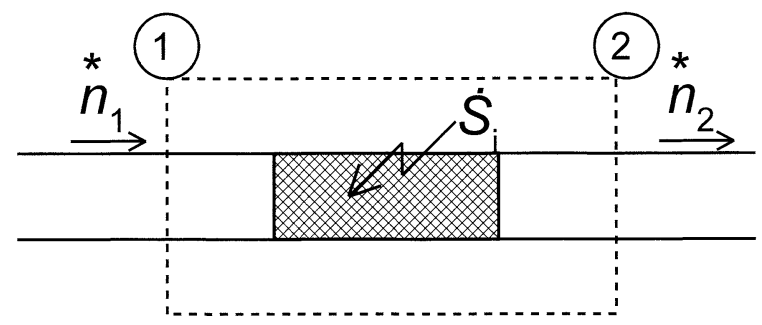

Fig. 6 Schematic diagram of the throttling process. The dotted line represents the (adiabatic) system. A molar flow $\stackrel{*}{n}{ }_{1}$ enters at position 1 and $\stackrel{*}{n_{2}}$ leaves at 2 
this may also be written as

$$
\dot{S}_{\mathrm{i}}=-\int_{1}^{2} \frac{*}{T} \mathrm{~d} p
$$

For small pressure drops, or in cases where $\stackrel{*}{V} / T$ is practically constant (as in liquid flow through a valve),

$$
\dot{S}_{\mathrm{i}}=\frac{*}{T}\left(p_{1}-p_{2}\right) .
$$

With the flow conductance $C$

$$
\stackrel{*}{V}=C\left(p_{1}-p_{2}\right)
$$

we get

$$
\dot{S}_{\mathrm{i}}=\frac{C\left(p_{1}-p_{2}\right)^{2}}{T} \text {. }
$$

\section{Ideal Regenerators and Heat Exchangers}

In this section we will discuss ideal regenerators and ideal heat exchangers.

\subsection{Ideal Regenerator}

An important component of refrigerators, operating with oscillatory flows, is the regenerator. A regenerator consists of a matrix of a solid porous material, such as spherical particles or metal sieves, through which gas flows, as shown in Fig. 7. The matrix must have a high heat capacity and a good heat contact with the gas. At the same time it should have a low flow resistance. These are conflicting requirements.

The thermodynamic and hydrodynamic properties of regenerators are extremely complicated, so one must make simplifying assumptions. The degree of idealization differs from case to case. In its most extreme form an ideal regenerator has the following properties:

1. the heat capacity of the matrix per unit volume is much larger than of the gas;

2. the heat contact between the gas and the matrix is perfect;

3 . the flow resistance of the matrix is zero;

Fig. 7 Schematic picture of a regenerator

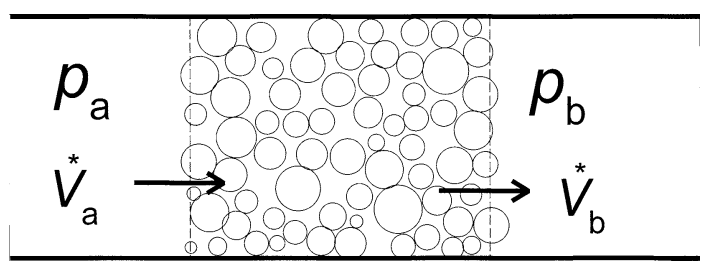


4. the porosity $g$, this is the fraction of the volume taken by the matrix material, is zero;

5. the thermal conductivity in the flow direction is zero;

6. the gas in the regenerator is ideal.

In an ideal regenerator the entropy production rate is zero. The average enthalpy flow in the regenerator is

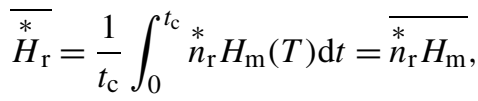

where $t_{\mathrm{c}}$ is the cycle time. The bar indicates time average. If the working fluid is an ideal gas then, with (175),

$$
\overline{H_{\mathrm{r}}}=C_{\mathrm{p}} \overline{n_{\mathrm{r}} T} .
$$

If conditions 1 and 2 are satisfied then the gas temperature varies with position but at a certain point it is constant in time so

$$
\overline{\stackrel{*}{H}_{\mathrm{r}}}=C_{\mathrm{p}} T \overline{*_{\mathrm{r}}} .
$$

In the steady state the time-average molar flow $\stackrel{*}{n}_{\mathrm{r}}$ in the regenerator is zero (in fact everywhere in the system) so

$$
\overline{n_{\mathrm{r}}}=0
$$

As a result of (54) and (55) the average enthalpy flow in an ideal regenerator is zero

$$
\overline{H_{\mathrm{r}}}=0 .
$$

The fact that the average enthalpy flow in an ideal regenerator with an ideal gas is zero implies that it has no cooling power. Any heat load on the regenerator has nowhere to go and can only lead to an increase of the local temperature. As we will see later, this differs for an ideal regenerator with a nonideal gas.

Depending on the situation one or more assumptions, which model the ideal regenerator, may be dropped. Usually they are replaced by other assumptions of a less rigorous nature. They will lead to nonzero enthalpy flow and entropy generation in the regenerator. This will be the topic of later sections.

The development of regenerator materials with a large heat capacity per $\mathrm{m}^{3}$ around $10 \mathrm{~K}[3]$ is one of the main reasons why the performance of cryocoolers is improved so much in recent times. A collection of specific heat data is found in Fig. 8 [4].

\subsection{Ideal Heat Exchanger}

An ideal heat exchanger has zero flow resistance and the temperature of the gas, leaving the heat exchanger, is equal to the (fixed) body temperature $T_{X}$ of the heat exchanger. However, even a perfect heat exchanger cannot affect the temperature $T_{\mathrm{i}}$ 


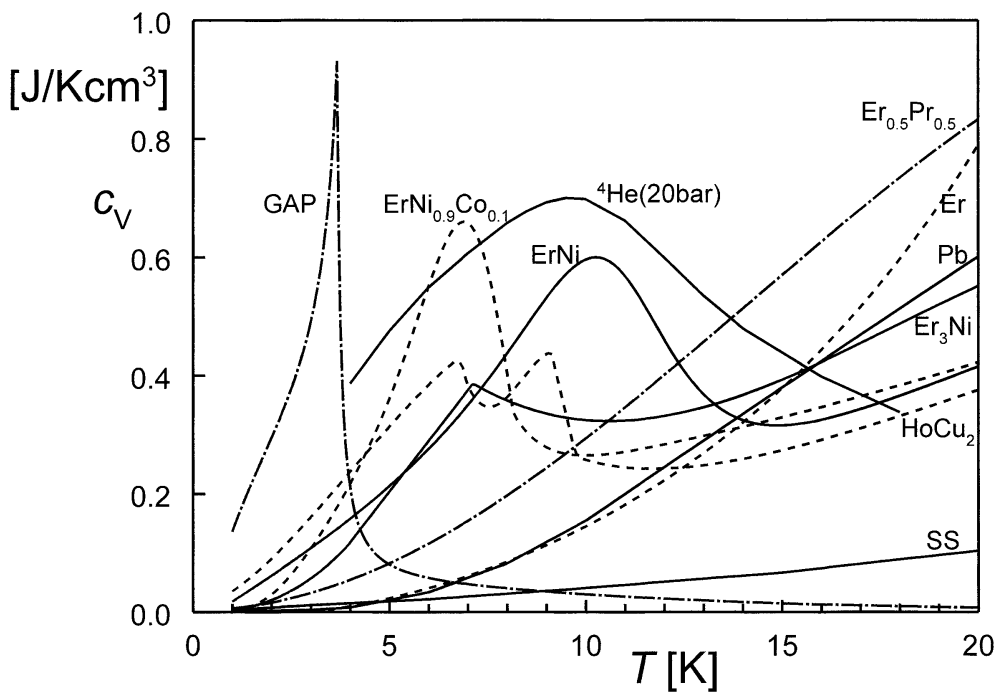

Fig. 8 Heat capacities per unit volume, $c_{\mathrm{V}}$, of various important regenerator materials as functions of temperature. (GAP is $\mathrm{Gd}_{2} \mathrm{AlO}_{3}$; $\mathrm{SS}$ is stainless steel)

of the incoming gas. This leads to a fundamental form of entropy production, which we now will calculate.

The heat transfer rate from the gas, with flow rate $\stackrel{*}{n}>0$ and with gas temperature $T$, to the heat-exchanger body, over a small section of the exchanger, is

$$
\delta \dot{Q}=-\stackrel{*}{n} C_{\mathrm{p}} \delta T
$$

with the associated entropy production rate, according to (38),

$$
\delta \dot{S}_{\mathrm{X}}=\delta \dot{Q}\left(\frac{1}{T_{\mathrm{X}}}-\frac{1}{T}\right)
$$

The total entropy production rate when the gas is cooled from $T_{\mathrm{i}}$ to $T_{\mathrm{X}}$ is

$$
\dot{S}_{\mathrm{X}}=\stackrel{*}{n} \int_{\mathrm{X}}^{\mathrm{i}}\left(\frac{1}{T_{\mathrm{X}}}-\frac{1}{T}\right) C_{\mathrm{p}} \mathrm{d} T .
$$

If $T_{\mathrm{i}} \approx T_{\mathrm{X}}$ this reduces to

$$
\dot{S}_{\mathrm{X}}={ }_{n}^{*} C_{\mathrm{p}} \frac{\left(T_{\mathrm{i}}-T_{\mathrm{X}}\right)^{2}}{2 T_{\mathrm{X}}^{2}} .
$$

Since both $T_{\mathrm{i}}-T_{\mathrm{X}}$ and $\stackrel{*}{n}$ are first-order terms the entropy production rate in the heat exchanger $\dot{S}_{\mathrm{X}}$ is of third-order and can be neglected in low-amplitude considerations. 


\section{entropy flows}

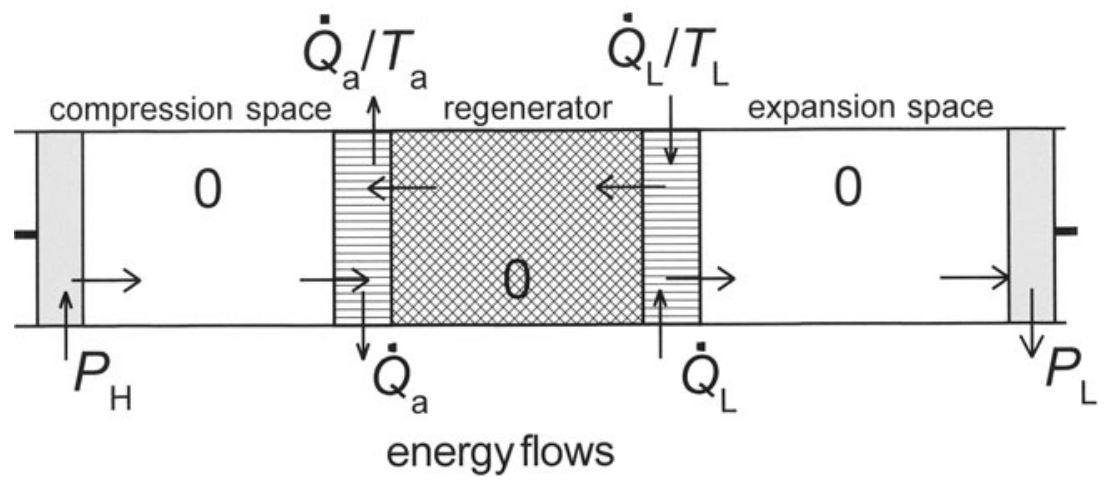

Fig. 9 Schematic diagram of a Stirling cooler. The system has one piston at ambient temperature $T_{\mathrm{a}}$ and one piston at low temperature $T_{\mathrm{L}}$. The upper half shows the entropy flows and the lower half the energy flows

\section{Stirling Refrigerators}

We will now turn to the treatment of the most important types of cryocoolers and related thermal machines. We will start with the Stirling-type refrigerators. The basic type of Stirling-type cooler is depicted in Fig. 9. At the left it consists of a piston, compression space, and heat exchanger, all at ambient temperature $T_{\mathrm{a}}$. Next comes a regenerator. At the right there are a heat exchanger, the expansion space, and a piston, all at the low temperature $T_{\mathrm{L}}$. The gas is compressed at ambient temperature and expanded at low temperature. The thermal contact with the surroundings, left and right, at the temperatures $T_{\mathrm{a}}$ and $T_{\mathrm{L}}$ is supposed to be so good that the compression and expansion are isothermal. The work, performed during the expansion, is recovered. The working fluid is helium.

The cooling cycle is divided in four steps as depicted in Fig. 10. We start a cycle when the two pistons are in their most left positions, the cold piston touches the cold heat exchanger:

1. From a to $b$. The warm piston moves to the right over a certain distance while the position of the cold piston is fixed. The compression at the hot end is isothermal by definition, so a certain amount of heat $Q_{\mathrm{a}}$ is given off to the surroundings at temperature $T_{\mathrm{a}}$.

2. From $\mathrm{b}$ to $\mathrm{c}$. Both pistons move to the right so that the volume between the two pistons remains constant. The gas enters the regenerator at the left with temperature $T_{\mathrm{a}}$ and leaves it at the right with temperature $T_{\mathrm{L}}$. During this part of the cycle heat is given off by the gas to the regenerator material. During this process the pressure drops and heat has to be supplied to the compression and expansion spaces to keep the temperatures constant.

3. From $\mathrm{c}$ to $\mathrm{d}$. The cold piston moves to the right while the position of the warm piston is fixed. The expansion is isothermal so heat $Q_{\mathrm{L}}$ is taken up from the application. 
Fig. 10 (Color online) Four states in the Stirling cycle

Fig. $11 p V$-diagram of the ideal Stirling cycle
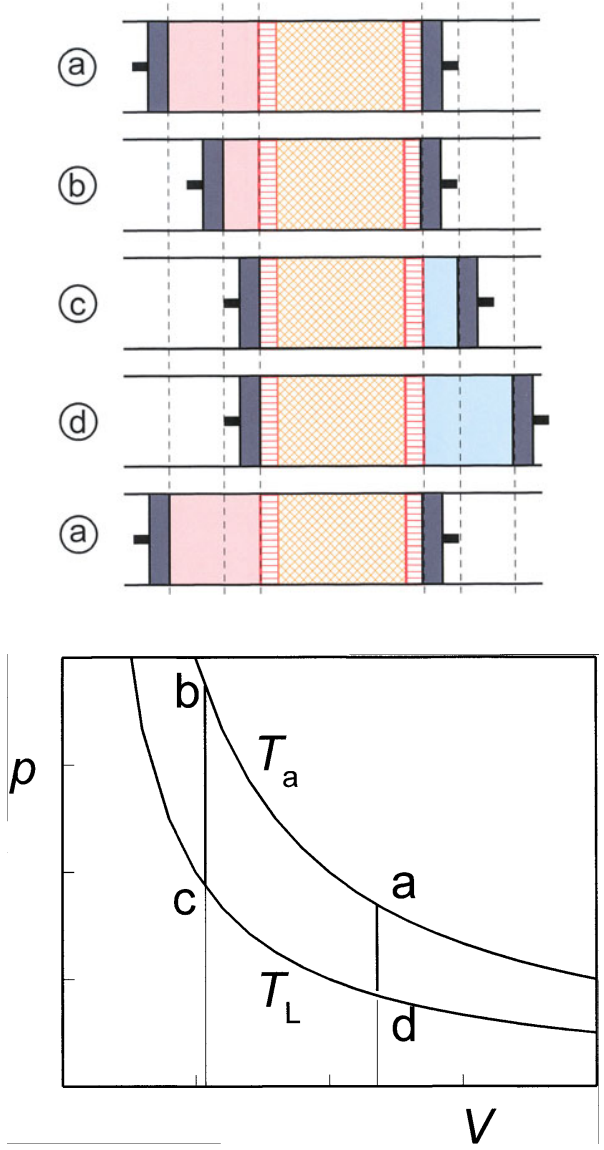

4. From d to a. Both pistons move to the left so that the total volume remains constant. The gas enters the regenerator at the right with temperature $T_{\mathrm{L}}$ and leaves it at the left with $T_{\mathrm{a}}$ so heat is taken up from the regenerator material. During this process the pressure increases and heat has to be extracted from the compression and expansion spaces to keep the temperatures constant. In the end of this step the state of the cooler is the same as at the start.

In the pV diagram (Fig. 11) the cycle consists of the well-known form of two isotherms and two isochores. The volume $V$ in this diagram is the volume between the two pistons. At each point of the cycle the pressure in the system and the volume are well-defined. However, during the steps 2 and 4 the temperature of part of the gas is $T_{\mathrm{a}}$ and of the other part it is $T_{\mathrm{L}}$.

In practice the cycle is not divided in discrete steps as described above. Usually the motion of both pistons are driven by a common rotary axes which makes the motions of the two pistons harmonic. It is typical for the Stirling cycle that the phase difference between the motion of the two pistons is about $90^{\circ}$.

The cycle is reversible and, with the surroundings of the cooler, heat is exchanged only at two fixed temperatures, so the COP is the Carnot COP given by (24). This 
can also be seen as follows. In Fig. 9 the entropy and energy flows are indicated. Based on the first law the power $P_{\mathrm{H}}$, supplied to the warm piston, is equal to the heat flow $\dot{Q}_{\mathrm{a}}$ to the surroundings, so $P_{\mathrm{H}}=\dot{Q}_{\mathrm{a}}$. The power $P_{\mathrm{L}}$, recovered at the cold piston, is equal to the cooling power $\dot{Q}_{\mathrm{L}}$, so $P_{\mathrm{L}}=\dot{Q}_{\mathrm{L}}$. The second law, applied to the regenerator and the two heat exchangers, gives

$$
\frac{\dot{Q}_{\mathrm{a}}}{T_{\mathrm{a}}}=\frac{\dot{Q}_{\mathrm{L}}}{T_{\mathrm{L}}} .
$$

If the power $P_{\mathrm{L}}$, released by the expansion, is used to reduce the net power $P$, supplied to the system, so $P=P_{\mathrm{H}}-P_{\mathrm{L}}$, this results in a $C O P$ of

$$
\xi=\frac{\dot{Q}_{\mathrm{L}}}{P_{\mathrm{H}}-P_{\mathrm{L}}}=\frac{T_{\mathrm{L}}}{T_{\mathrm{a}}-T_{\mathrm{L}}} .
$$

The cold piston, as described above, is rather impractical, so, in many cases, the cold expander is avoided by using a displacer. A displacer is a solid body which moves back and forth and drives the gas back and forth between the warm and the cold end of the system through the regenerator. Ideally the pressure over the displacer is zero, so no work is required to move it. Its motion is synchronized with the motion of the piston. Typically it is ninety degrees out of phase. Also in this case the cycle is reversible and heat is exchanged, with the surroundings, only at two fixed temperatures, so the efficiency is also the Carnot efficiency given by (24).

Another type of Stirling cooler is the split-pair type [5] as shown in Fig. 12. It consists of a compressor, a split pipe, and a cold finger. Usually the compressor is a linear compressor where the piston is driven by an AC magnetic field as in loudspeakers. There are often two pistons which move in opposite directions to reduce mechanical vibrations. The pistons are suspended by so-called flexure bearings which provide

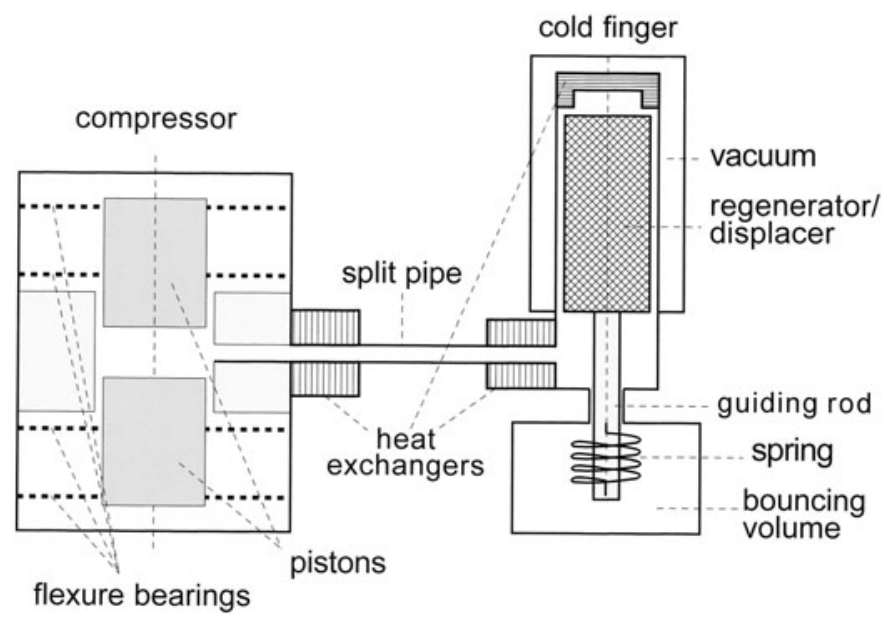

Fig. 12 Schematic diagram of a split-pair Stirling refrigerator. The cooling power is supplied to the heat exchanger of the cold finger. Usually the heat flows are so small that there is no need for physical heat exchangers around the split pipe 
stiffness in the radial direction and flexibility in the axial directions. The piston and the compressor casing don't touch so no lubricants are needed.

In the cold finger the moving part is the regenerator which works as the displacer at the same time. It is suspended by a spring which can also be a flexure bearing. The motion of the displacer/regenerator is driven by the pressure drop between the upper space (expansion space) and the lower space and by the pressure difference between the bouncing volume and the cold-finger volume. Hence, the area of the cross section of the guiding rod is an important design parameter. The cooler operates at a frequency near the resonance frequency of the mass-spring system inside the cold finger. The motions of the piston and the displacer/regenerator are similar to the motions of the displacer-type Stirling cooler.

\section{GM-Refrigerators}

We will now describe the workhorse of many low-temperature systems: the GiffordMcMahon (GM) cooler [6]. GM coolers are robust machines that find wide-spread application e.g. in MRI and cryopumping. Figure 13 is a schematic diagram. The working fluid is helium at pressures in the 10 to 30 bar range. The cold head contains a regenerator and a displacer which usually are combined in one body. The varying pressure is obtained by connecting the cold head periodically to the high- and lowpressure sides of a compressor through a rotating valve which is synchronized with the motion of the displacer.

During the opening and closing of the valves irreversible processes take place, so GM-coolers are intrinsically irreversible. This is a clear disadvantage of this type of machine. On the other hand the cycle frequencies of the compressor and the displacer are uncoupled. E.g. the compressor runs at power-line frequency while the cycle of the cold head is $1 \mathrm{~Hz}$. This means that the swept volume of the compressor can be 50(60) times smaller than of the cooler. Basically cheap compressors of domestic refrigerators can be used, but special precautions have to be taken to prevent overheating of the compressor since they are not designed for helium. In addition very high quality purification traps have to be installed to prevent oil vapor from entering the regenerator.

The cycle can be divided in four steps, with Fig. 14, as follows: The starting position is with the low-pressure valve closed, the high-pressure valve open, and the

Fig. 13 Schematic diagram of a GM-cooler. $V_{1}$ and $V_{\mathrm{h}}$ are buffer volumes of the compressor. The compression heat is removed by the cooling water of the compressor via a heat exchanger. The rotary valves alternatingly connect the cooler to the high- and the low-pressure sides of the compressor and runs synchronous with the displacer

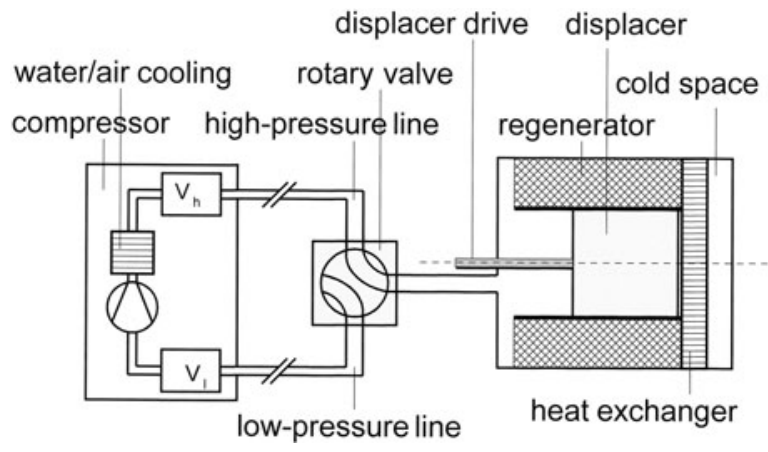


Fig. 14 The four stages in the cooling cycle of the GM cooler
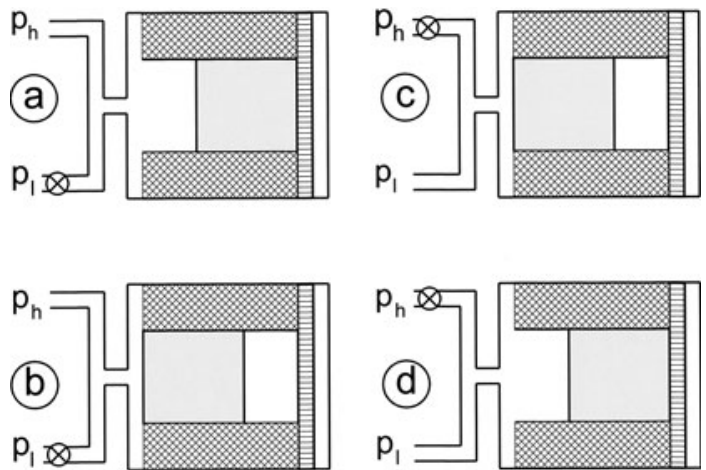

displacer all the way to the right (so in the cold region). All the gas is at room temperature.

1. From a to b. The displacer moves to the left while the cold head is connected to the high-pressure side of the compressor. The gas is forced to pass the regenerator. It enters the regenerator at ambient temperature $T_{\mathrm{a}}$ and leaves it with temperature $T_{\mathrm{L}}$ so heat is given off by the gas to the regenerator material. Due to the high density of the low-temperature gas some additional gas will flow from the highpressure side of the compressor through the regenerator to the low-temperature space.

2. From b to c. The cold head is connected to the low-pressure side of the compressor with fixed position of the displacer. Part of the gas flows through the regenerator to the low-pressure side of the compressor. Expansion of the gas takes place. The expansion in the cold space is isothermal so heat is taken up from the application. During this phase of the cycle the useful cooling power is produced.

3. From $\mathrm{c}$ to $\mathrm{d}$. The displacer moves to the right while the cold head is still connected to the low-pressure side of the compressor forcing the cold gas to pass the regenerator, while taking up heat from the regenerator.

4. From d to a. The cold head is connected to the high-pressure side of the compressor with fixed position of the displacer. In the end of this step the cycle is closed.

The ideal cooling power can be obtained immediately from the first law, applied to the expansion space with volume $V_{\mathrm{e}}$ and pressure $p_{\mathrm{e}}$

$$
\dot{Q}_{\mathrm{L}}=\overline{p_{\mathrm{e}} \frac{\mathrm{d} V_{\mathrm{e}}}{\mathrm{d} t}} .
$$

We have seen before that the average enthalpy flow, in an ideal regenerator, is zero. The energy $\dot{Q}_{\mathrm{L}}$ leaves the cold end of the system as enthalpy transported by the displacer.

The flow distribution in regenerators is homogeneous. As a result the cooling power tends to be proportional to the area of the cross section of the regenerator. This is illustrated in Fig. 15 where the length of GM coolers and the external diameter of their heat exchangers are plotted as functions of the square root of the 
Fig. 15 Length $L$ and diameter $D$ of the GM-coolers of Cryomech plotted as functions of $\sqrt{\dot{Q}}$ at $80 \mathrm{~K}[7]$

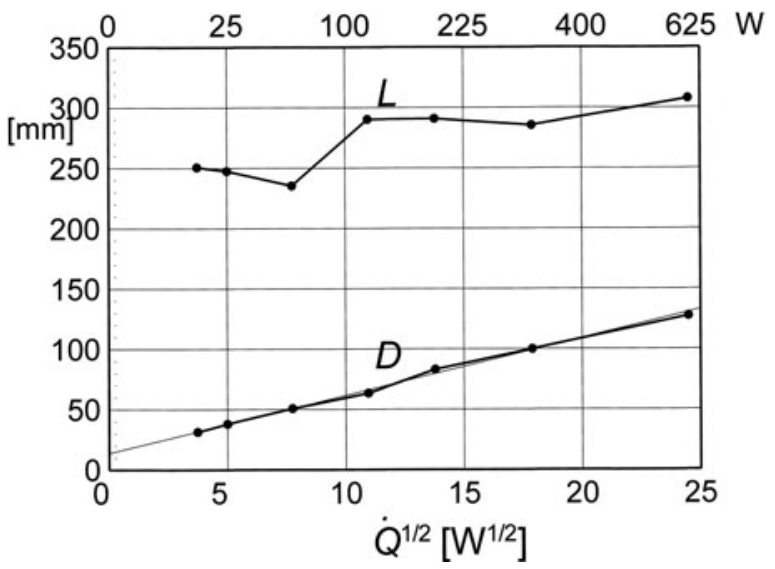

cooling power at $80 \mathrm{~K}, \dot{Q}^{1 / 2}$ [7]. The length is practically constant and the diameter (minus $13 \mathrm{~mm}$ ) is nicely proportional to, $\dot{Q}^{1 / 2}$. So, increasing the cooling power is simply a matter of increasing the diameters of the cooler components. However, instabilities were found in regenerators with a large diameter/length ratio [8].

\section{Pulse-Tube Refrigerators}

\subsection{Components of PTR's}

This section gives a description of the basic operation of a Pulse-Tube Refrigerator (PTR) in the steady state. ${ }^{2}$ This type of cooler was invented by Mikulin [11]. At the moment there are many variants of PTR's. A so-called Stirling-type single-orifice PTR is represented schematically in Fig. 16. From left to right it consists of:

1. A piston which moves back and forth.

2. A heat exchanger $\mathrm{X}_{1}$ (after cooler) where heat is released at room temperature $\left(T_{\mathrm{a}}\right)$ to cooling water or to the surroundings.

3. A regenerator.

4. A heat exchanger $\mathrm{X}_{\mathrm{L}}$ at low temperature $\left(T_{\mathrm{L}}\right)$ where heat is absorbed from the application.

5. A tube, often called the pulse tube.

6. A heat exchanger $\mathrm{X}_{3}$ to room temperature $\left(T_{\mathrm{a}}\right)$.

7. A flow resistance (orifice).

8. A buffer volume, in which the pressure $p_{\mathrm{B}}$ is practically constant.

In this section all flow resistances are neglected except from the orifice. The flow conductance $C$ of the orifice is adjusted for optimum performance. Typically the

\footnotetext{
${ }^{2}$ The operation of this system should not be confused with the operation of the so-called basic pulse tube whose operation is based on a heat shuttle interaction with the tube wall $[9,10]$.
} 


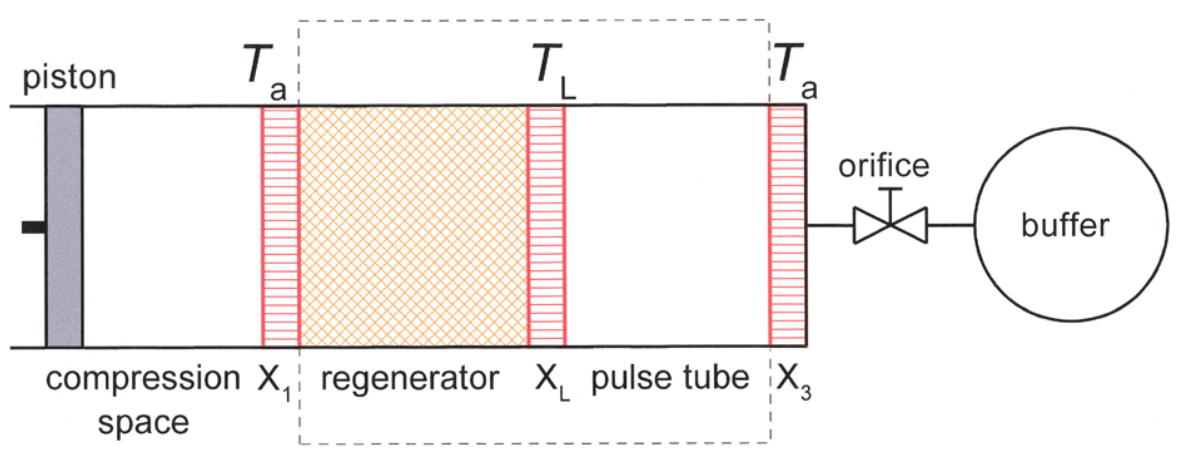

Fig. 16 (Color online) Schematic diagram of a Stirling-type single-orifice PTR. From left to right: piston, after cooler $\left(\mathrm{X}_{1}\right)$, regenerator, low-temperature heat exchanger $\left(\mathrm{X}_{\mathrm{L}}\right)$, tube (pulse tube), second room-temperature heat exchanger $\left(\mathrm{X}_{3}\right)$, orifice $(\mathrm{O})$, buffer. The dotted rectangle represents thermal (vacuum) insulation

dimensionless flow resistance of the orifice $\alpha$, defined by

$$
\alpha=\frac{\omega V_{\mathrm{t}}}{C \gamma p_{0}},
$$

is of order one. Here $\omega$ is the angular frequency and $p_{0}$ the average pressure. The system is filled with helium at $p_{0}$ of typically 20 bar. The part in between the heat exchangers $\mathrm{X}_{1}$ and $\mathrm{X}_{3}$ is below room temperature.

\subsection{Cooling Principle}

Driven by the piston, the gas moves back and forth and the pressure $p_{\mathrm{t}}$ in the pulse tube varies. The operation frequency is typically 1 to $50 \mathrm{~Hz}$ and the working fluid is helium at, say, 10 to 30 bar. The pressure varies smoothly. Acoustic effects, such as traveling pressure waves, or fast pressure changes, are absent. In the regenerator and in heat exchangers the gas is in good thermal contact with its surroundings while in the pulse tube the gas is thermally isolated. In the pulse tube compression of gas leads to heating and expansion to cooling.

In and around the pulse tube we can distinguish three types of gas parcels. There are gas parcels that, during a cycle, move in and out the pulse tube via the cold heat exchanger $\mathrm{X}_{\mathrm{L}}$, gas parcels that move in and out via the hot heat exchanger $\mathrm{X}_{3}$, and gas parcels that never leave the pulse tube. Together the latter form the so-called gas piston. Schematic drawings of the temperature-position curves of these three types of gas parcels are given in Fig. 17. At the hot end gas flows from the buffer via the orifice into the tube with a temperature $T_{\mathrm{a}}$ if $p_{\mathrm{t}}<p_{\mathrm{B}}$. If $p_{\mathrm{t}}=p_{\mathrm{B}}$ the gas at the hot end stops and if $p_{\mathrm{t}}>p_{\mathrm{B}}$ the gas moves back towards the hot end of the tube and, eventually, through the heat exchanger $\mathrm{X}_{3}$ and the orifice into the buffer. So gas elements enter the pulse tube if $p_{\mathrm{t}}<p_{\mathrm{B}}$ and leave it if $p_{\mathrm{t}}>p_{\mathrm{B}}$. Consequently, at the hot end, the gas leaves the tube with a temperature higher than the inlet temperature $T_{\mathrm{a}}$ and heat is released via the heat exchanger $\mathrm{X}_{3}$ to the surroundings.

At the cold end the analysis is a bit more complicated due to the fact that the velocity $v_{\mathrm{L}}$ at the cold end is determined by the velocity $v_{\mathrm{H}}$ of the gas at the hot end 
Fig. 17 Left side: a gas element enters the tube at temperature $T_{\mathrm{L}}$ and leaves it at a lower temperature hence producing cooling. Right side: a gas element enters the tube at temperature $T_{\mathrm{a}}$ and leaves it at a higher temperature hence producing heating. Middle: this gas element is in the gas piston and never leaves the pulse tube. It moves to the right with high $T$ and to the left with low $T$

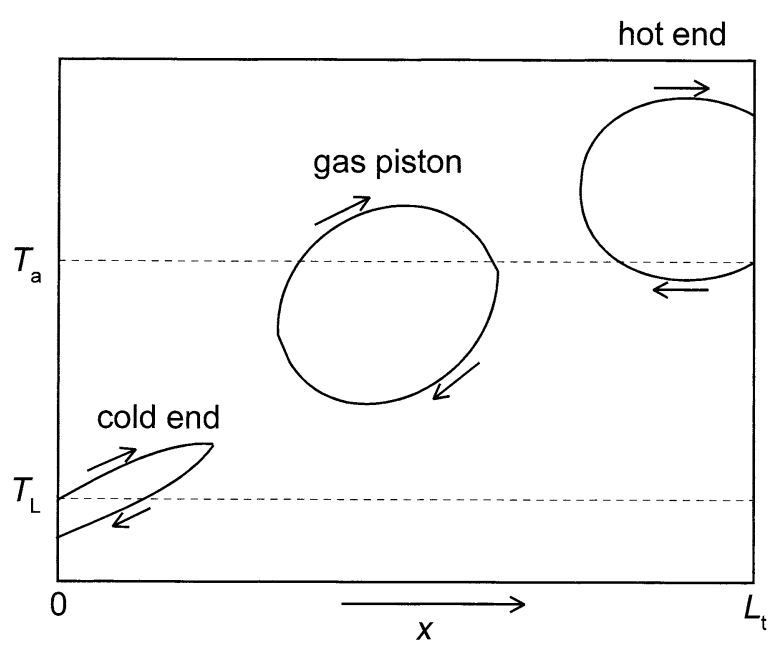

and by the elasticity of the gas column in the tube, according to (33),

$$
v_{\mathrm{L}}=v_{\mathrm{H}}+\frac{V_{\mathrm{t}}}{\gamma A_{\mathrm{t}} p_{\mathrm{t}}} \frac{\mathrm{d} p_{\mathrm{t}}}{\mathrm{d} t}
$$

with $V_{\mathrm{t}}$ and $A_{\mathrm{t}}$ the volume and area of the pulse tube respectively. Still the situation at the cold end is basically the same as at the hot end. At the cold end the gas enters the tube with temperature $T_{\mathrm{L}}$ when the pressure is high. It returns to $\mathrm{X}_{\mathrm{L}}$ when the pressure is low and the temperature is below $T_{\mathrm{L}}$. Hence producing cooling.

Also gas parcels in the gas piston move to the right with a high temperature and back with a low temperature. As we will see in the next section this is reason why there is a net enthalpy flow in the pulse tube.

\subsection{Thermodynamics of PTR's}

In this Subsection we will analyze the PTR based on the first and second law of thermodynamics. In the ideal case entropy is produced only in the orifice. In all the other subsystems $\dot{S}_{\mathrm{i}}=0$. The heat flows $\dot{Q}$ with the surroundings are nonzero only in the heat exchangers. Flows to the right are counted positive. The signs of the power and the heat flows are defined in Fig. 18. We split up the PTR in subsystems going from right to left. First we consider the simple system represented in Fig. 19a, containing only the orifice. At the reservoir side the pressure $p=p_{\mathrm{B}}$ and the temperature $T=T_{\mathrm{a}}$ are constant. As a result the molar entropy $S_{\mathrm{m}}$ and the molar enthalpy $H_{\mathrm{m}}$ are constant as well. Similar to (55) it follows that the average enthalpy and entropy flows in the tube, connecting the orifice with the buffer, are zero. In the tube, connecting the orifice and the heat exchanger $\mathrm{X}_{3}$, the temperature is constant as well so the average enthalpy flow here is also zero. The first law for the orifice shows that the heat flow into the orifice, needed to keep its temperature constant, is zero as well. 


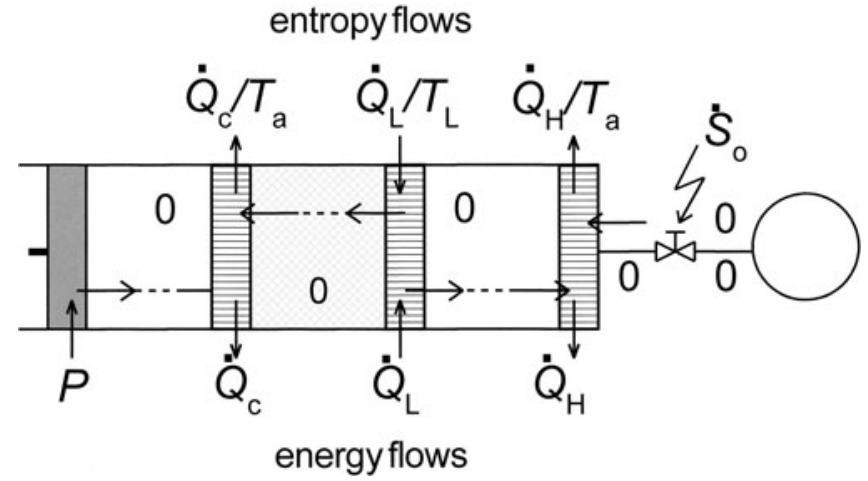

Fig. 18 Schematic diagram of a single-orifice PTR. In the upper half of the figure the entropy flows are indicated. The lower half gives the energy flows. If a particular flow is zero in a certain region this is indicated by ' 0 '. It is assumed that the PTR is ideal so that dissipation only takes place in the orifice
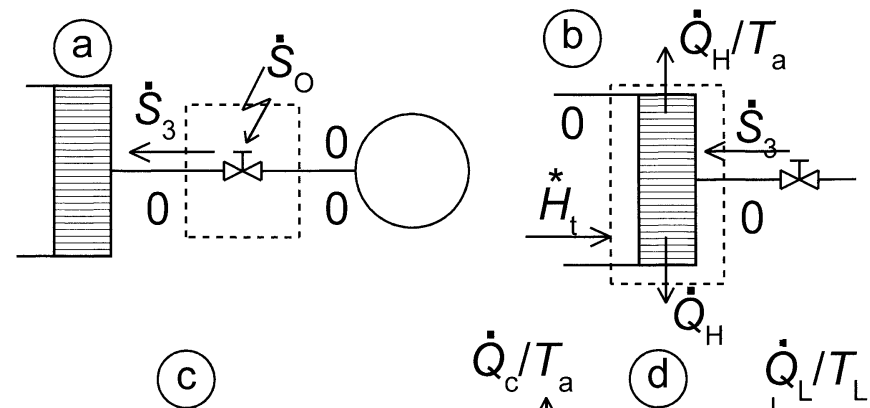

$\dot{Q}_{\mathrm{c}} / T_{\mathrm{a}}$
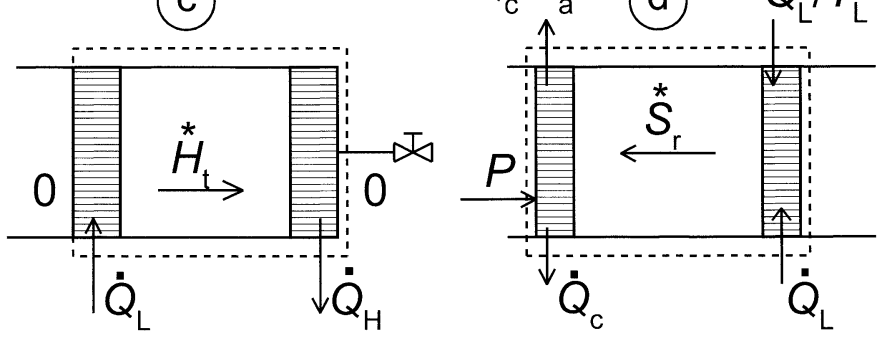

Fig. 19 Thermodynamic systems containing the orifice (a), the heat the exchanger $X_{3}(\mathbf{b})$, the pulse tube and its heat exchangers (c), and the regenerator and its heat exchangers (d)

The average entropy flow in the tube between the heat exchanger $\mathrm{X}_{3}$ and the orifice $\stackrel{*}{S}_{3}$ is nonzero due to the pressure variations. With (173) we may write it as

$$
\overline{S_{3}}=-R \bar{n} \overline{\ln \frac{p_{\mathrm{t}}}{p_{0}}} .
$$

Since the heat flow to the orifice is zero the entropy production rate in the orifice is

$$
\overline{\dot{S}_{\mathrm{O}}}=-\stackrel{*}{S}_{3} \text {. }
$$


So the entropy, which is produced in the orifice, flows towards $\mathrm{X}_{3}$ as shown in Fig. 19a.

Next we consider the exchanger $X_{3}$ as a thermodynamic system (Fig. 19b). In the pulse tube the gas moves back and forth isentropically, so the average entropy flow here is zero ${ }^{3}$

$$
\overline{S_{\mathrm{t}}}=0 .
$$

The second law with (67) gives that the average heat flow, extracted at $\mathrm{X}_{3}$, is given by

$$
\dot{Q}_{\mathrm{H}}=T_{\mathrm{a}} \overline{\dot{S}_{\mathrm{O}}}
$$

So heat has to be extracted at $\mathrm{X}_{3}$. From the first law this must be equal to the average enthalpy flow in the pulse tube

$$
\overline{H_{\mathrm{t}}}=\dot{Q}_{\mathrm{H}} .
$$

It is interesting to note that the entropy flow to system b comes from the right and the enthalpy flow from the left.

Now we consider the system containing the tube and its two heat exchangers (Fig. 19c). The average enthalpy flow in the pulse tube is given by

$$
\overline{H_{\mathrm{t}}}=C_{\mathrm{p}} \bar{*} T \text {. }
$$

The gas moves to the right with a high temperature and to the left with a low temperature (see the gas parcel in the gas piston in Fig. 17). As a result the net enthalpy transport in the pulse tube $\stackrel{*}{H}_{\mathrm{t}}$ is nonzero. However, the enthalpy flows at the left and right of system $\mathrm{c}$ are zero (see (56)). The first law then gives that

$$
\dot{Q}_{\mathrm{L}}=\dot{Q}_{\mathrm{H}}
$$

This important relation shows that the cooling power is equal to the heat released at the warm heat exchanger $\mathrm{X}_{3}$. It seems to contradict the Clausius formulation of the second law applied to the system Fig. 19c. However, the Clausius principle only applies to closed systems.

The second law, applied to $\mathrm{X}_{\mathrm{L}}$, with (68) gives

$$
\dot{Q}_{\mathrm{L}}=-T_{\mathrm{L}}{\stackrel{*}{S_{\mathrm{r}}}}
$$

where $\stackrel{*}{S}_{\mathrm{r}}$ is the entropy flow in the regenerator. The negative sign means that the average entropy flow in the regenerator is directed from the cold to the warm end.

\footnotetext{
${ }^{3}$ That the entropy flow is zero is not obvious for surfaces in the neighborhood of one of the heat exchangers since gas parcels can exchange heat with the heat exchanger when they move back and forth. This problem can be solved by starting to make up the balance of the entropy, transported through the surface in a cycle, when the first parcel moves into the pulse tube. All parcels passing the surface will return with the same entropy.
} 
This is logical since the gas moves to the right when the pressure is high (low entropy, see (173)) and to the left when the pressure is low (entropy high). In the ideal case there is no entropy production in the regenerator so the average entropy flow

$$
\overline{S_{\mathrm{r}}}=\text { constant. }
$$

Now consider a system consisting of the regenerator and its two adjacent heat exchangers $\mathrm{X}_{1}$ and $\mathrm{X}_{\mathrm{L}}$ as in Fig. 19d. The gas in the (adiabatic) compression space moves back and forth isentropically, so the average entropy flow left of the after cooler $\mathrm{X}_{1}$ is zero. Combining (73) and (74) gives

$$
\frac{\dot{Q}_{\mathrm{L}}}{T_{\mathrm{L}}}=\frac{\dot{Q}_{\mathrm{c}}}{T_{\mathrm{a}}} .
$$

The first law requires that the average heat release at the aftercooler

$$
\dot{Q}_{\mathrm{c}}=P .
$$

Here $P$ is the power input in the compressor. The $C O P$ (see (22)) is found by combining (75) and (76) which gives the well known [12] result

$$
\xi=\frac{T_{\mathrm{L}}}{T_{\mathrm{a}}}
$$

Comparing this value with the Carnot COP, given by (24), shows that the efficiency of an ideal PTR is less than of an ideal cooler. This is due to the dissipation in the orifice. Close to room temperature the difference between $\xi$ and $\xi_{\mathrm{C}}$ is big, but at temperatures in the range of liquid nitrogen $(77 \mathrm{~K})$ and lower the difference in $C O P$ is insignificant for most applications.

Combining (69) and (72) shows that

$$
\dot{Q}_{\mathrm{L}}=T_{\mathrm{a}} \overline{\dot{S}_{\mathrm{O}}}
$$

So the cooling power is determined by the dissipation at the hot end of the tube which, in turn, is determined by the component of the oscillating flow which is in phase with the pressure. The flow at the cold end contains a term which is not in phase with the pressure variations (see (65)). This component is bad for the efficiency as it adds to the dissipation in the regenerator but not to the cooling power. Furthermore the outof-phase component of the gas flow is a load on the regenerator. Most of the research and development work in PTS's aimed at suppressing the out-of-phase flow at the cold end of the pulse tube. The phase difference between the pressure and the flow at the cold end can be controlled with proper devices such as: double inlet, inertance, four-valve, active buffer, and warm expander [13-16].

\subsection{The Various Types of PTR}

So far we have discussed the Stirling-type single-orifice PTR (Fig. 16). The pressure variations are generated by a compressor which is directly connected to the 


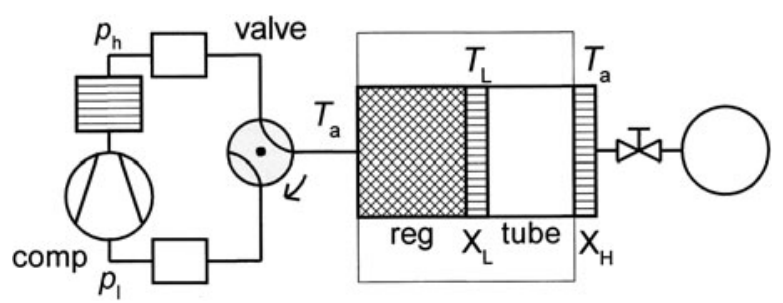

Fig. 20 Schematic diagram of a GM-type PTR. The compressor delivers constant pressure levels $p_{1}$ and $p_{\mathrm{h}}$. The varying pressure in the cooler unit is obtained through a rotating valve which alternatingly connects the regenerator to the low- and high-pressure sides of the compressor

cold head. Typical operation frequencies are 20 to $50 \mathrm{~Hz}$ and the temperature range $50 \mathrm{~K}$ and higher. However, for getting cooling, the source of the pressure variations is unimportant. PTR's for lower temperatures (20 K and below) usually operate at low frequencies ( 1 to $2 \mathrm{~Hz}$ ) and with pressure variations from 10 to $25 \mathrm{bar}$ (pressure ratio 2.5). At room temperature the swept volume per cycle would be very high (up to one liter and more). Therefore the compressor is uncoupled from the cooler just like in the GM-coolers. A schematic diagram is given in Fig. 20. A system of valves is needed, which alternatingly connects the high pressure and the low pressure to the hot end of the regenerator. Usually this is a rotating valve. The high-temperature part at the compressor side is the same as in GM-coolers (see Fig. 13). Therefore, this type of PTR is called a GM-type PTR. The gas flows through the valves are accompanied by losses which are absent in the Stirling-type PTR.

PTR's can be classified according to their shape. If the regenerator and the tube are in line (as in Figs. 16 and 20) we talk about a linear PTR. The disadvantage of the linear PTR is that the cold spot is in the middle of the cooler. For many applications it is preferable that the cooling is produced at the end of the cooler. By bending the PTR we get a U-shaped cooler as shown in Fig. 21. Both hot ends can be mounted on the flange of the vacuum chamber at room temperature. This is the most common shape of PTR's. For some applications it is preferable to have a cylindrical geometry. In that case the PTR can be constructed in a coaxial way so that the regenerator becomes a ring-shaped space surrounding the tube.

The lowest temperature, reached with single stage PTR's, is just above $10 \mathrm{~K}$ [17]. However, one PTR can be used to precool the other as in Fig. 21. Note that the hot end of the second tube is connected to room temperature and not to the cold end of the first stage. In this clever way it is avoided that the heat, released at the hot end of the second tube, is a load on the first stage. In applications the first stage also operates as a temperature-anchoring platform for e.g. shield cooling of superconducting-magnet cryostats. Matsubara and Gao were the first to cool below $4 \mathrm{~K}$ with a three-stage PTR [18]. With two-stage PTR's temperatures of $2.1 \mathrm{~K}$, so close to the $\lambda$-point of helium, have been obtained. With a three-stage PTR $1.73 \mathrm{~K}$ has been reached using ${ }^{3} \mathrm{He}$ as the working fluid [19].

It is also possible to couple two independent PTR's where one precools the other. In these systems there can be no uncontrolled internal circulations (DC-flow) of the 
Fig. 21 Two-stage, U-shaped, GM-type, double-orifice PTR. The first stage precools the second one. Note that the hot end of the second stage is connected to room temperature and not to the cold end of the first stage. The so-called minor orifices, which suppress a possible DC flow, are not shown

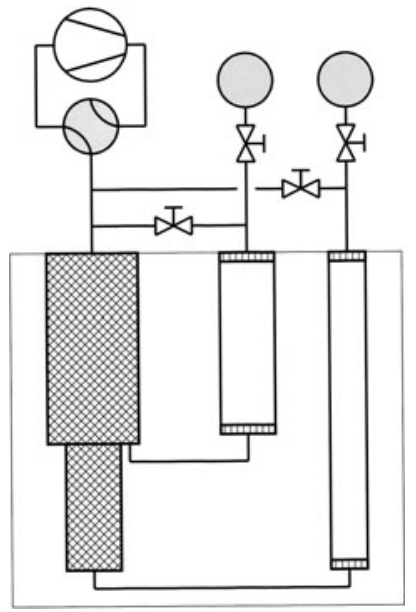

gas and each of the PTR's can be optimized separately. In this kind of system the world record of low temperature in PTR's $(1.27 \mathrm{~K})$ is reached while the second system was operated with ${ }^{3} \mathrm{He}$ as the working fluid [20]. With a superfluid vortex cooler, precooled by this system, the temperature has been lowered to $1.19 \mathrm{~K}$ with the potential of obtaining $0.7 \mathrm{~K}$ in this way [21]. For lower temperatures one needs ${ }^{3} \mathrm{He}$ cryostats or dilution refrigerators which will be discussed later.

\section{Real Regenerators}

Cryocoolers can reach temperatures below $20 \mathrm{~K}$ where helium is not an ideal gas. Furthermore ideal regenerators, as described above, do not exist in practice. For the most general case numerical models must be used [22-24]. In this Section we will discuss rather realistic regenerators, but there will be still some idealizations. We will limit the discussion to the linear approximation, using the harmonic model. In the harmonic approximation regenerators can be mathematically described by the transfer matrix. This takes into account the viscosity of the working fluid, the porosity of the matrix, the ratio of the two end temperatures, the hydraulic diameter of the pores, the length and cross section of the regenerator, the frequency of the oscillations, and the gas pressure as in (211) and following [25].

\subsection{Temperature Variations}

An expression for the temperature variation of the gas is derived in Ref. [26]. Neglecting the heat conduction term (this can be treated separately) Equation (31) in Ref. [26] reads

$$
\frac{g C_{\mathrm{p}}}{V_{\mathrm{m}}} \frac{\partial T_{\mathrm{g}}}{\partial t}=-\frac{*_{\mathrm{r}}}{A_{\mathrm{r}}}\left(C_{\mathrm{p}} \frac{\partial T_{\mathrm{g}}}{\partial l}+H_{\mathrm{p}} \frac{\partial p}{\partial l}\right)+g T_{\mathrm{g}} \alpha_{\mathrm{V}} \frac{\partial p}{\partial t}+\beta\left(T_{\mathrm{r}}-T_{\mathrm{g}}\right) .
$$


Here $g$ is the porosity (void fraction) of the matrix, $H_{\mathrm{p}}$ is defined by (165), $\beta$ is the heat-exchange coefficient per unit volume, and $\alpha_{\mathrm{V}}$ the volumetric expansion coefficient (see (167)). The first term in the right-hand side is the convective contribution, the second term is due to the compression of the gas (see (162)), and the last term due to heat flow from the matrix to the gas. In complex form, using that the $\partial p / \partial l$-term would give a second-order contribution,

$$
\mathrm{i} \omega \frac{g C_{\mathrm{p}}}{V_{\mathrm{m} 0}} \hat{T}_{\mathrm{g}}=-\frac{\hat{n}_{\mathrm{r}} C_{\mathrm{p}}}{A_{\mathrm{r}}} \frac{\mathrm{d} T_{0}}{\mathrm{~d} l}+\mathrm{i} \omega g T_{0} \alpha_{\mathrm{V} 0} \hat{p}-\beta\left(\hat{T}_{\mathrm{g}}-\hat{T}_{\mathrm{r}}\right) .
$$

The index 0 indicates time-averaged values.

For the matrix temperature, again neglecting heat conduction,

$$
(1-g) c_{\mathrm{V}} \frac{\partial T_{\mathrm{r}}}{\partial t}=\beta\left(T_{\mathrm{g}}-T_{\mathrm{r}}\right)
$$

where $c_{\mathrm{V}}$ the heat capacity per unit volume of the bulk regenerator matrix material as given in Fig. 8. Equation (81), in complex form, gives

$$
\hat{T}_{\mathrm{r}}=\frac{\beta}{\mathrm{i} \omega(1-g) c_{\mathrm{V} 0}+\beta} \hat{T}_{\mathrm{g}}
$$

Equations (80) and (82) can be used to calculate the enthalpy flow in the regenerator. Together with the heat conduction, and requiring that the total average energy flow is constant, the temperature profile of the regenerator can be calculated.

\subsection{Nonideal-Gas Effects in PTR's}

\subsubsection{Introduction}

In Sect. 3 it was shown that an ideal regenerator, with an ideal gas, has no cooling power i.e. heat, applied somewhere to the regenerator, will lead to an unlimited increase of the local temperature. This is different if the working fluid is a real gas. In this Section, we will consider the situation that heat $\dot{Q}_{\mathrm{r}}(l)$ is supplied externally to the regenerator as shown in Fig. 22.

In the nonideal-gas effect the pressure dependent contribution of the enthalpy, $H_{\mathrm{p}}$ in (164), plays a key role. The $H_{\mathrm{p}}$ values for ${ }^{4} \mathrm{He}$ can be obtained from Ref. [27] and for ${ }^{3} \mathrm{He}$ from Refs. [28, 29]. Examples at 15 bar are given in Fig. 23. For a Van

Fig. 22 Schematic drawing of the regenerator with a heat load $\mathrm{d} \dot{Q}_{\mathrm{r}} / \mathrm{d} l$ per unit length, distributed along the matrix, and a cooling power $\dot{Q}_{\mathrm{L}}$ at the cold end

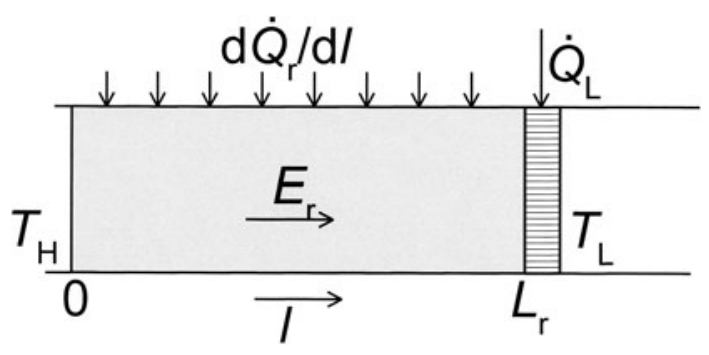


Fig. 23 Plots of $H_{\mathrm{p}}$ at 15 bar for ${ }^{4} \mathrm{He}$ and ${ }^{3} \mathrm{He}$ as functions of temperature

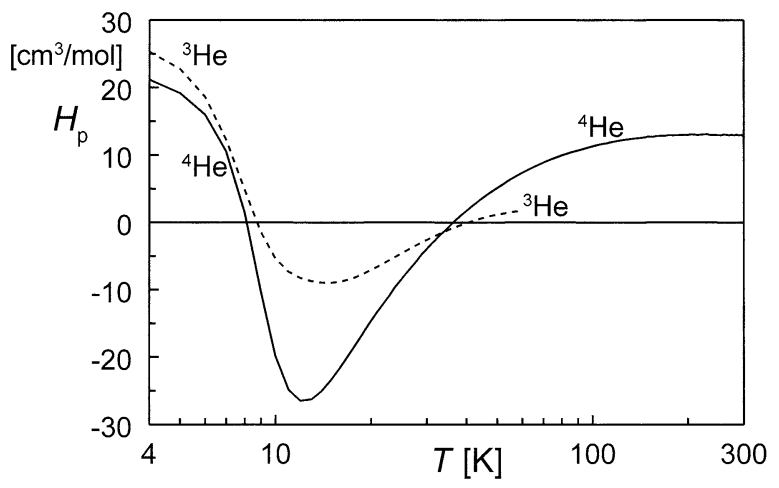

der Waals gas the values of $H_{\mathrm{p}}$ at $T=0$ and $T=\infty$ are the same and equal to the parameter $b$, the total volume of the atoms. Figure 23 also shows that helium is not an ideal gas $\left(H_{\mathrm{p}} \neq 0\right)$ even at the high temperatures.

Since nonideal-gas effects are most relevant in the second stage of a PTR we will consider the regenerator of the second stage of a PTR. It will be treated as ideal. However, it cannot be assumed that the thermal conductivity in the flow direction is zero since without heat flow energy conservation cannot be satisfied. But we will assume that the flow resistance and the void volume are zero, that the thermal contact between the gas and the matrix is perfect, and that the heat capacity of the matrix is very big. Furthermore we assume that the pressure variations are harmonic

$$
p=p_{0}+p_{\mathrm{A}} \cos \omega t
$$

with $p_{\mathrm{A}} \ll p_{0}$. As the flow resistance is neglected $p_{\mathrm{A}}$ is constant. Finally we assume that the molar flow is in phase with the pressure and given by

$$
\stackrel{*}{n_{\mathrm{r}}}=n_{\mathrm{A}} \cos \omega t
$$

As the void volume of the regenerator is zero $n_{\mathrm{A}}$ is constant. That the flow is in phase with the pressure can be achieved by proper flow-controlling devices at the hot end of the second-stage pulse tube. The expressions, in the following subsections, will be treated to lowest relevant order. This means that pressure and temperature changes will be treated in first order, and energy-related quantities such as enthalpy flow and cooling powers to second order. The discussion in this section regards PTR's, but can also be applied to other types of coolers.

\subsubsection{Cooling Power}

The index $\mathrm{L}$ will be used to indicate values at the cold end of the regenerator. The cooling power of the second stage can be calculated from the first law, applied to $\mathrm{X}_{\mathrm{L}}$,

$$
0=\dot{Q}_{\mathrm{cL}}+\dot{Q}_{\mathrm{L}}+\overline{H_{\mathrm{rL}}}-\overline{H_{\mathrm{t}}} .
$$


Here $\dot{Q}_{\mathrm{cL}}$ is the heat flow on $\mathrm{X}_{\mathrm{L}}$ by conduction via the regenerator, $\dot{Q}_{\mathrm{L}}$ the applied

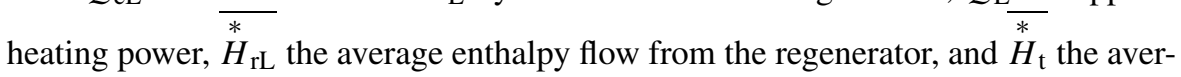
age enthalpy flow in the pulse tube. At the regenerator side of $\mathrm{X}_{\mathrm{L}}$ the temperature is constant so, using (164),

$$
\overline{H_{\mathrm{rL}}}=H_{\mathrm{pL}} \bar{*} \delta p
$$

At the pulse-tube side the entropy is constant so (163) gives

$$
\overline{H_{\mathrm{t}}}=V_{\mathrm{mL}} \bar{*} \delta p
$$

Combining (85), (86), and (87) gives

$$
\dot{Q}_{\mathrm{L}}=\left(V_{\mathrm{mL}}-H_{\mathrm{pL}}\right) \stackrel{\bar{*} n \delta p}{n}-\dot{Q}_{\mathrm{cL}}
$$

With (83), (84), and (166) we get

$$
\dot{Q}_{\mathrm{L}}=\frac{1}{2} n_{\mathrm{A}} p_{\mathrm{A}} V_{\mathrm{mL}} T_{\mathrm{L}} \alpha_{\mathrm{VL}}-\dot{Q}_{\mathrm{cL}} .
$$

With the volume-flow amplitude

$$
U_{\mathrm{A}}=V_{\mathrm{mL}} n_{\mathrm{A}}
$$

we get

$$
\dot{Q}_{\mathrm{L}}=\frac{1}{2} U_{\mathrm{A}} p_{\mathrm{A}} T_{\mathrm{L}} \alpha_{\mathrm{VL}}-\dot{Q}_{\mathrm{cL}} .
$$

So the cooling power depends on $\alpha_{\mathrm{VL}}$. Even if $\dot{Q}_{\mathrm{cL}}=0$ the cooling power $\dot{Q}_{\mathrm{L}}$ is zero if $\alpha_{\mathrm{VL}}=0$. So the lowest temperature, that can be reached with PTR's, is the temperature where $\alpha_{\mathrm{V}}=0$. For ${ }^{4} \mathrm{He}$ this is just above the lambda point and for ${ }^{3} \mathrm{He}$ at $15 \mathrm{bar}$ it is $1.04 \mathrm{~K}$ [29]. The lowest temperature, reached in experiment, is $1.27 \mathrm{~K}$, [20] so very close to the theoretical minimum.

In Ref. [30] it is derived that the $C O P$ of an ideal PTR, for $T_{\mathrm{L}}$ below about $7 \mathrm{~K}$, is given by

$$
\xi=\frac{V_{\mathrm{mL}} \alpha_{\mathrm{VL}}}{V_{\mathrm{mH}}} T_{\mathrm{L}} .
$$

Equation (92) shows that the COP is determined by $V_{\mathrm{mL}} \alpha_{\mathrm{VL}}$, which is larger for ${ }^{3} \mathrm{He}$ than for ${ }^{4} \mathrm{He}$ (see Fig. 24). Also the difference in thermal conductivity and viscosity of ${ }^{3} \mathrm{He}$ and ${ }^{4} \mathrm{He}$ plays a role.

So far we focused on the regenerator. One may wonder how the real-gas effect affects the energy flow in the pulse tube. The answer is in (87) which can also be written as

$$
\overline{H_{\mathrm{t}}}=\stackrel{\bar{*} V_{\mathrm{L}} \delta p}{ }
$$


Fig. 24 The product $V_{\mathrm{m}} \alpha_{\mathrm{V}}$ at 15 bar for ${ }^{3} \mathrm{He}$ and ${ }^{4} \mathrm{He}$ as functions of $T$

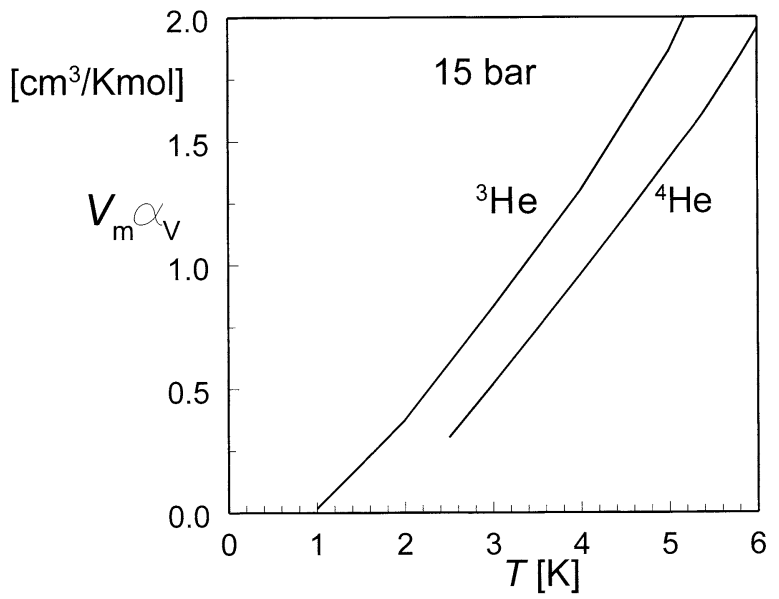

The values of $\stackrel{*}{V} \delta p$ at the cold and the hot end of the pulse tube are the same, so the enthalpy flow in the pulse tube is unaffected by the fact that we are dealing with a nonideal gas.

\subsubsection{Temperature Profile in the Regenerator}

In the previous subsection we have seen that the cooling power of PTR's is reduced due to real-gas effects. Fortunately some of this cooling power can be recovered by using the cooling power of the regenerator which is nonzero in the case of real gases. This can be understood as follows: the average total energy flow $E_{\mathrm{r}}$ in the regenerator is the sum of the heat flow

$$
\dot{Q}_{\mathrm{c}}=-A_{\mathrm{r}} \kappa_{\mathrm{r}} \frac{\mathrm{d} T}{\mathrm{~d} l},
$$

(with $\kappa_{\mathrm{r}}$ the thermal conductivity of the regenerator) and the average enthalpy flow, so

$$
E_{\mathrm{r}}=\dot{Q}_{\mathrm{c}}+\overline{H_{\mathrm{r}}}
$$

If $\dot{Q}_{\mathrm{r}}(l)$ is the total amount of heat, supplied externally to the regenerator between 0 and $l$, energy conservation requires that

$$
E_{\mathrm{r}}(l)=E_{\mathrm{r}}(0)+\dot{Q}_{\mathrm{r}}(l) .
$$

With (164) isothermal enthalpy changes, as in our ideal regenerator, are equal to $\delta H_{\mathrm{m}}=H_{\mathrm{p}} \delta p$, so, with (83) and (84) the time average enthalpy flow in the regenerator is given by

$$
\overline{*_{\mathrm{r}}}=\frac{1}{2} n_{\mathrm{A}} p_{\mathrm{A}} H_{\mathrm{p}} .
$$


Fig. 25 Temperature profiles in case heat is supplied at the middle of the regenerator. The heating powers are indicated in watt at the corresponding curves

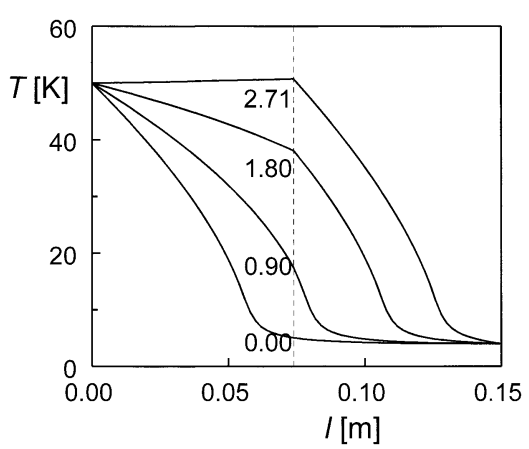

Due to our assumptions of zero flow resistance and zero void volume, $n_{\mathrm{A}} p_{\mathrm{A}}$ does not depend on $l$. These relations result in

$$
A_{\mathrm{r}} \kappa_{\mathrm{r}} \frac{\mathrm{d} T}{\mathrm{~d} l}=\frac{1}{2} n_{\mathrm{A}} p_{\mathrm{A}} H_{\mathrm{p}}(T)-E_{\mathrm{r}}(0)-\dot{Q}_{\mathrm{r}}(l) .
$$

The heat input per unit length $\mathrm{d} \dot{Q}_{\mathrm{r}}(l) / \mathrm{d} l$ can be distributed evenly e.g. if the regenerator is used to precool an incoming flow of ${ }^{4} \mathrm{He}$ (as in Ref. [31]) or ${ }^{3} \mathrm{He}$ (as in Fig. 36). The heat input can also be discrete (as in Refs. [32, 33]). We will consider here the case where heat is supplied only in the middle of the regenerator (so at $\left.l=L_{\mathrm{r}} / 2\right)$. Integration of (98) is performed numerically. The values of $T_{\mathrm{L}}$ and $T_{\mathrm{H}}$ are fixed (here at 4 and $50 \mathrm{~K}$ respectively) and, by iteration, the value of $E_{\mathrm{r}}(0)$, which satisfies this boundary condition, is determined. Next the temperature profile is obtained from (98). In our calculations we take $L_{\mathrm{r}}=0.15 \mathrm{~m}, A_{\mathrm{r}}=20 \mathrm{~cm}^{2}$, and the operating frequency $v=2 \mathrm{~Hz}$ in a PTR with $p_{0}=15$ bar. The effective thermal conductivity is taken as $\kappa_{\mathrm{r}}=2.5 \mathrm{~W} / \mathrm{Km}$ and $\frac{1}{2} n_{\mathrm{A}} p_{\mathrm{A}}=0.13 \mathrm{~mol} \mathrm{MPa} / \mathrm{s}$.

Figure 25 gives the temperature profiles in the regenerator for four values of the heating power $\dot{Q}_{\mathrm{r}}$ supplied in the middle of the regenerator. For zero $\dot{Q}_{\mathrm{r}}$ the temperature profile is rather flat at the low-temperature end. So $\dot{Q}_{\mathrm{cL}}$ is practically zero and the load on the $\mathrm{X}_{\mathrm{L}}$ from the regenerator side is mainly due to enthalpy flow. The temperature $T_{\mathrm{i}}$ of the point where the heat is supplied increases with $\dot{Q}_{\mathrm{r}}$ and a kink in the $T$-profile appears. Figure 26 gives plots of $\dot{Q}_{\mathrm{L}}$ and $T_{\mathrm{i}}$ as functions of $\dot{Q}_{\mathrm{r}}$. At low $\dot{Q}_{\mathrm{r}}$ values the $\dot{Q}_{\mathrm{L}}$ remains fairly constant (hence "free" cooling power) at a value of about $0.42 \mathrm{~W}$. For $\dot{Q}_{\mathrm{r}}>2.7 \mathrm{~W} \dot{Q}_{\mathrm{L}}$ is negative, which means that the temperature of $4 \mathrm{~K}$ cannot be maintained without external cooling.

\section{Thermoacoustics}

The field of thermoacoustics is pioneered by Rott [34] and by Swift [35] and his coworkers. See Ref. [36] and the references therein. In order to thoroughly understand thermoacoustic machines it is important to understand sound in terms of temperatureposition variations rather than the usual pressure-velocity variations. Therefore this Section starts with a description of sound in these terms. In ordinary speech the sound intensity is $65 \mathrm{~dB}$ which corresponds with pressure variations of $51 \mathrm{mPa}$, displacements of $0.2 \mu \mathrm{m}$, and temperature variations of $43 \mu \mathrm{K}$. In thermoacoustic systems, 
Fig. 26 The cooling power $\dot{Q}_{\mathrm{L}}$ and the temperature $T_{\mathrm{i}}$ as functions of $\dot{Q}_{\mathrm{r}}$. The dotted lines represent the temperatures of the hot and the cold ends

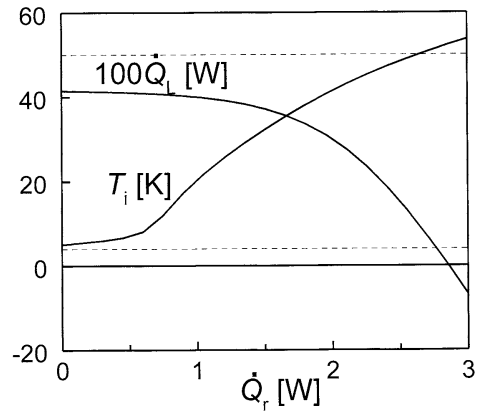

with sound levels of $180 \mathrm{~dB}$, the pressure variations are $0.3 \mathrm{bar}$, displacements more than $10 \mathrm{~cm}$, and the temperature variations $24 \mathrm{~K}$. Although a pressure ratio of $30 \%$ is very big for acoustic systems, this is still much smaller than the pressure ratios obtained e.g. in GM-type refrigerators, where pressure variations of a factor 3 are common.

\subsection{Sound}

The wave equation in one dimension reads

$$
\frac{\partial^{2} v}{\partial x^{2}}-\frac{\partial^{2} v}{c^{2} \partial t^{2}}=0
$$

with $v$ the gas velocity and $c$ the sound velocity satisfying

$$
c^{2}=\gamma \frac{p_{0}}{\rho_{0}} .
$$

For an ideal gas

$$
c^{2}=\gamma \frac{R T_{0}}{M},
$$

with $M$ the molar mass. In these expressions $p_{0}, T_{0}$, and $\rho_{0}$ are the average pressure, temperature, and density respectively. Important special cases of solutions of (99) are monochromatic plane waves which are superpositions of traveling waves to the right and to the left

$$
v=v_{\mathrm{Ar}} \cos \left(\varphi_{\mathrm{r}}-k(x-c t)\right)+v_{\mathrm{Al}} \cos \left(\varphi_{1}+k(x+c t)\right) .
$$

By proper shifts in the $t$ - and $x$-axis and with

$$
\omega=k c
$$

this can be put in a simpler form

$$
v=v_{\mathrm{Ar}} \cos (\omega t-k x)+v_{\mathrm{Al}} \cos (\omega t+k x) .
$$


The pressure variations are given by

$$
\delta p=c \rho_{0}\left[v_{\mathrm{Ar}} \cos (\omega t-k x)-v_{\mathrm{Al}} \cos (\omega t+k x)\right] .
$$

The deviation $\delta x$ of the gas-particle position from its equilibrium position $x$ can be obtained by integration of (104) and is given by

$$
\delta x=\frac{v_{\mathrm{Ar}}}{\omega} \sin (\omega t-k x)+\frac{v_{\mathrm{Al}}}{\omega} \sin (\omega t+k x) .
$$

With (105) and (169) the temperature variations are

$$
\delta T=\frac{c M}{C_{\mathrm{p}}}\left[v_{\mathrm{Ar}} \cos (\omega t-k x)-v_{\mathrm{Al}} \cos (\omega t+k x)\right] .
$$

Equations (106) and (107) form a parametric representation of a tilted ellipse in the $\delta T-\delta x$ plane with $t$ as the parameter. If $v_{\mathrm{Ar}}=v_{\mathrm{Al}}$ we have a pure standing wave and the ellipse of the $\delta T-\delta x$ plot is reduced to a straight line with slope

$$
\frac{\mathrm{d} \delta T}{\mathrm{~d} \delta x}=\frac{\omega c M}{C_{\mathrm{p}}} \tan (k x) .
$$

Figure $27 \mathrm{a}$ is a graph of the position and the velocity amplitude of a pure standing wave in a half-wavelength tube together with the plot of the pressure and temperature amplitudes. At the tube ends the displacement is zero while the temperature variation is maximal, so the $\delta T-\delta x$ plot is a vertical line here. All points, representing the $\delta T-$ $\delta x$ in time, move back and forth over the lines, as shown in Fig. 27b, so the average enthalpy transport, by a standing wave, is zero.

If we would put a thin horizontal metal plate (with negligible longitudinal heat conduction) in the sound field the thermal interaction of the moving gas with the plate leads to the thermoacoustic effects. The interaction with the gas would lead to a temperature profile in the plate which exactly matches the lines in Fig. 27b which have the so-called critical temperature gradients. If we would force (by heat exchangers) the actual temperature gradient in the plate to be smaller than the local slopes of

Fig. 27 a: Plot of the amplitudes of the velocity and displacements, and the pressure and temperature variations in a half-wavelength tube of a pure standing wave. b: corresponding $\delta T-\delta x$ plots of a standing wave. c: $\delta T-\delta x$ plots of a pure traveling wave
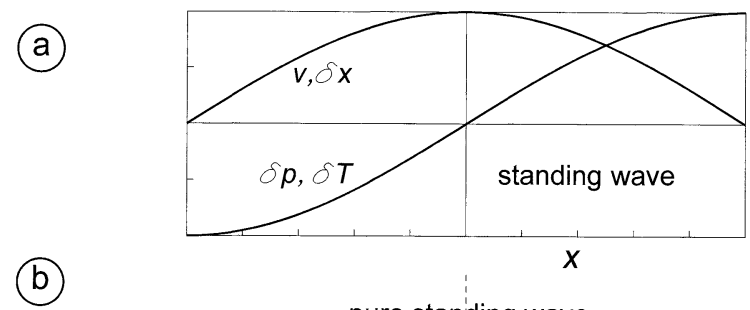

(b)

(C)

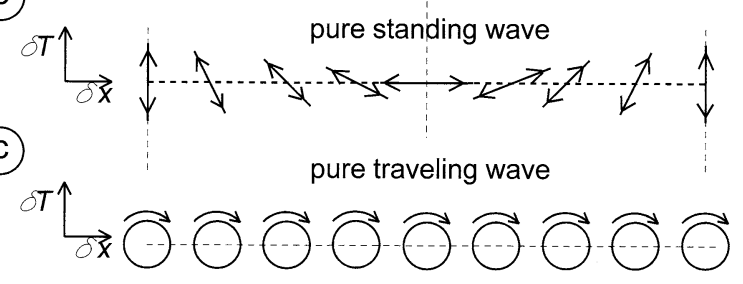


the $\delta T-\delta x$ plots, due to heat exchange with the surroundings, we have a cooler (or heat pump). If the temperature gradient in the plate is forced to be larger then we have an engine.

If $v_{\mathrm{Al}}=0$ or $v_{\mathrm{Ar}}=0$ we have pure traveling waves. With the proper scales of the axis, (106) and (107) represent circles in the $\delta T-\delta x$ diagram. Figure 27c represents the $\delta T-\delta x$ plots of a pure traveling wave to the right. The gas elements move to the right with high temperature and back with a low temperature, so there is a net transport of enthalpy. It is interesting to compare the $\delta T-\delta x$ plots with similar plots in the pulse tube of a PTR (Fig. 17).

\subsection{Energy Transport by Sound}

Sound can transport energy. In general the enthalpy flow is given by (2). With the molar flow

$$
\stackrel{*}{n}=\frac{\stackrel{*}{V}}{V_{\mathrm{m} 0}}=\frac{p_{0}}{R T_{0}} \stackrel{*}{V}
$$

and the volume flow

$$
\stackrel{*}{V}=A v
$$

with $A$ the tube cross section, and (175) we get for the average enthalpy flow

$$
\stackrel{*}{H}=\frac{p_{0} C_{\mathrm{p}}}{R T_{0}} A \overline{v \delta T} .
$$

As the expansion and compression are isentropic we use (169) to get the well-known relation of the enthalpy flow, which is also the acoustic energy flow

$$
\bar{*}+A \overline{v \delta p} \text {. }
$$

Note that this relation only holds for small amplitudes. Therefore, in general, (112) cannot be used to calculate the energy transport in the pulse tube of a PTR.

The velocity is given by (104) and the temperature by (107) so substitution in (111) gives

$$
\bar{H}=\gamma \frac{p_{0}}{2 c} A\left(v_{\mathrm{Ar}}^{2}-v_{\mathrm{Al}}^{2}\right) .
$$

This elegant relation shows that the enthalpy flow is independent of the $x$-coordinate (which is a consequence of energy conservation) and proportional to the difference in the velocity amplitudes squared of the positive and the negative sound wave.

\subsection{Thermoacoustic Systems}

Perhaps the simplest type of thermoacoustic device (from the point of view of construction) is depicted in Fig. 28a. It represents a so-called thermoacoustic prime mover. Typically it is $20 \mathrm{~cm}$ long with two cm diameter. Another important component is the so-called stack. Usually this is a stack of metal plates (stainless steel) at 
Fig. 28 a: Schematic diagram of a thermoacoustic prime mover; b: schematic diagram of a thermoacoustic refrigerator

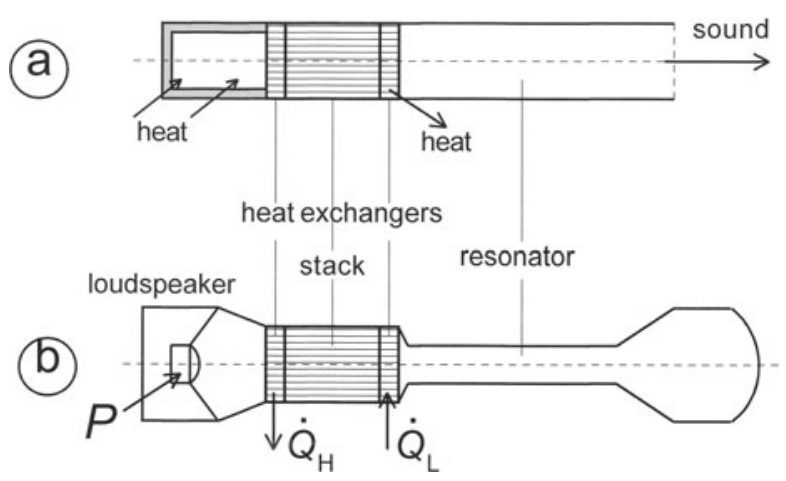

a mutual distance $2 y_{0}$ which is several times the thermal penetration depth $\delta_{\kappa}$ of the gas. The stack can also be a plug of loosely packed stainless steel wool or screens. There is also a heat exchanger to ambient temperature and a resonator tube. It is heated at the left e.g. by a propane flame. If the temperature at the hot side is high enough the system produces a loud sound.

Figure $28 \mathrm{~b}$ represents a thermoacoustic refrigerator. It is similar to the prime mover, but it has a driver (loudspeaker) at the left. The systems of Fig. 28a and b are so-called quarter-wavelength tubes which means that they resonate at a frequency so that the length of the tube is equal to a quarter of the wavelength of the sound. The quarter wavelength corresponds with the left half of Fig. 28a,b where the slopes of the $\delta T-\delta x$ lines of the standing wave are negative. In other words: the left side of a horizontal plate in the sound field is hotter than the right side. Although the conus of the loudspeaker in Fig. 28b is moving it is close to a velocity node, so its amplitude is small.

The operation of standing-wave systems relies on the time delay between gas transport and heat transport. They are in essence irreversible machines, so they have an inherently reduced efficiency. The mathematical description of the operation of these so-called standing-wave systems is more complicated than of traveling-wave systems. Therefore we will start with the discussion of traveling-wave systems.

\subsubsection{Traveling-Wave Systems}

This subsection treats the basics of so-called traveling-wave thermoacoustic engines in general terms. The engine, described in the paper by Backhaus and Swift [38], can serve as a model system. Figure 29a is a schematic drawing. It consists of a resonator tube and a loop which contains a regenerator, several heat exchangers, a compliance, a connecting tube, a pulse tube (also called thermal buffer tube), and a section, with a smaller diameter, called the inertance. As the energy flow in the regenerator is small (ideally it is zero), the main energy flow in the loop is from the hot heat exchanger via the pulse tube, the inertance, and to the main heat exchanger. This can be considered as energy transport via a traveling wave as in Fig. 27c. Hence the name travelingwave systems.

One of the fascinating properties of thermoacoustic systems is that they produce sound if the hot side is hot enough. Reference [39] describes transient effects theoretically. The set of first-order differential equations, which describes the dynamics 

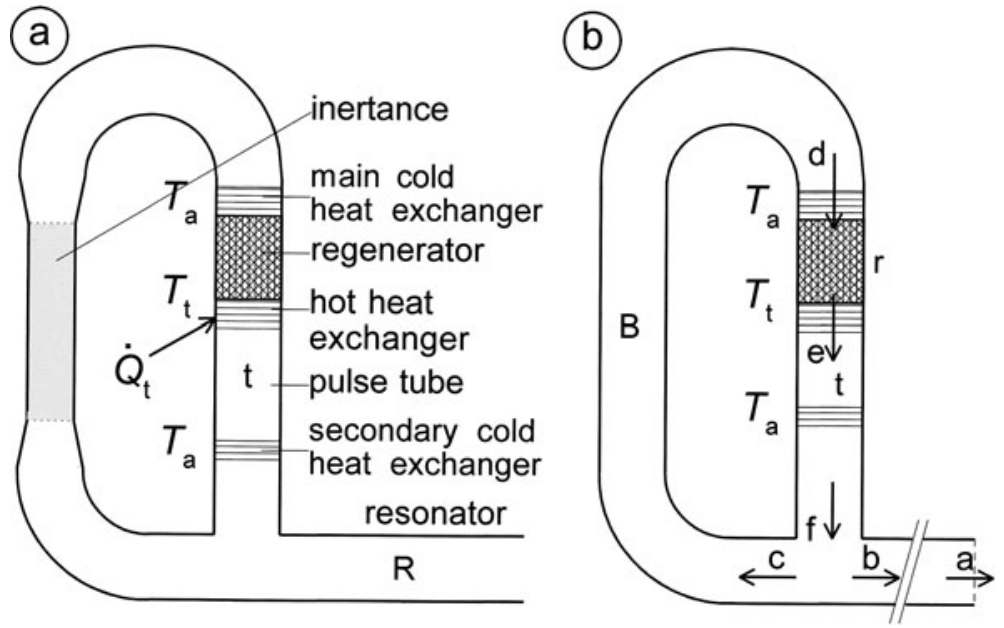

Fig. 29 (a) Active end of the thermoacoustic engine. The symbols are explained in the text. (b) Model system. a, b, c, d, e, and $\mathrm{f}$ are position indicators. The arrows define the directions of positive flow

of the individual components, is combined to a single high-order (fourth-order) differential equation which determines the time dependences of all dynamic variables. It is subsequently solved analytically. Unfortunately the full mathematical treatment is rather elaborate, even for a simplified model, and beyond the scope of this paper. Here only a description will be given of the basic principles.

The gas in the system starts to oscillate "spontaneously" with a certain angular frequency $\omega$ if the temperature $T_{\mathrm{t}}$ of the hot heat exchanger is above an onset temperature $T_{\mathrm{O}}$. If $T_{\mathrm{t}}<T_{\mathrm{O}}$ oscillations in the system die out with a certain decay time. If $T_{\mathrm{t}}$ is increased the decay time becomes longer and longer. If $T_{\mathrm{t}}>T_{\mathrm{O}}$ the oscillations grow. Only if $T_{\mathrm{t}}=T_{\mathrm{O}}$ there are steady oscillations. The amplitude is determined by the heating power $\dot{Q}_{\mathrm{t}}$ applied to the hot heat exchanger (minus the heat loss due to conduction to the surroundings). In principle $T_{\mathrm{t}}=T_{\mathrm{O}}$, independent of $\dot{Q}_{\mathrm{t}}$ if $\dot{Q}_{\mathrm{t}}$ is high enough. On the way to the steady state spectacular transient effects are possible, with temperature overshoots and bursts of high-intensity sound, which are not systematically studied so far.

As thermoacoustic systems tend to oscillate at their resonance frequency they have to be big to keep the frequency low. There are several interesting idea's to reduce the frequency by introducing solid [40] or liquid masses [41]. By doing so thermoacoustic systems merge with the more classical type of Stirling systems as the free-piston Stirling machines discussed above.

Now we turn to the steady-state operation and apply the harmonic model to derive the onset temperature and operation frequency. Our model system is depicted in Fig. 29b and consists of an open resonator tube $\mathrm{R}$ and a loop containing a bypass tube $\mathrm{B}$, a regenerator $\mathrm{r}$, and a pulse-tube section $\mathrm{t}$. It is assumed that all tubes have the same cross sectional area $A$. So there is no section in the loop with a smaller diameter (inertance) nor sections with a large diameter (compliance) [42]. The temperatures are assumed to be close to ambient temperature $T_{\mathrm{a}}$ except for inside and near the regenerator. Dissipative effects are neglected everywhere except in the regenerator. 
For the bypass channel B, with length $L_{\mathrm{B}}$, holds (225)

$$
\left(\begin{array}{c}
\hat{p}_{\mathrm{c}} \\
\hat{U}_{\mathrm{c}}
\end{array}\right)=\boldsymbol{T}_{\mathrm{B}}\left(\begin{array}{c}
\hat{p}_{\mathrm{d}} \\
\hat{U}_{\mathrm{d}}
\end{array}\right)
$$

with

$$
\boldsymbol{T}_{\mathrm{B}}=\left(\begin{array}{cc}
c_{\mathrm{B}} & \mathrm{i} \frac{c \rho_{0}}{A} s_{\mathrm{B}} \\
\mathrm{i} \frac{A}{c \rho_{0}} s_{\mathrm{B}} & c_{\mathrm{B}}
\end{array}\right)
$$

with $c$ the sound velocity and $\rho_{0}$ the gas density at $T_{\mathrm{a}}$. In order to get compact expressions $c_{\mathrm{B}}$ is used for $\cos \left(k L_{\mathrm{B}}\right)$ and $s_{\mathrm{B}}$ for $\sin \left(k L_{\mathrm{B}}\right)$ (with $k$ given by (103)) and a similar notation for the pulse tube (label $t$ ) and the resonator tube (label $\mathrm{R}$ ). By replacing the index B by $t$ or $\mathrm{R}$ the transfer matrices of the pulse tube $\boldsymbol{T}_{\mathrm{t}}$ and the resonator tube $\boldsymbol{T}_{\mathrm{R}}$ are obtained respectively. For the regenerator, with assumed (real) flow resistance $R_{\mathrm{r}}$ and zero porosity, holds (207)

$$
\left(\begin{array}{c}
\hat{p}_{\mathrm{d}} \\
\hat{U}_{\mathrm{d}}
\end{array}\right)=\boldsymbol{T}_{\mathrm{r}}\left(\begin{array}{l}
\hat{p}_{\mathrm{e}} \\
\hat{U}_{\mathrm{e}}
\end{array}\right)
$$

with

$$
\boldsymbol{T}_{\mathrm{r}}=\left(\begin{array}{cc}
1 & R_{\mathrm{r}} \\
0 & \frac{T_{\mathrm{a}}}{T_{\mathrm{t}}}
\end{array}\right)
$$

For the loop c, d, e, f we write

$$
\left(\begin{array}{c}
\hat{p}_{\mathrm{c}} \\
\hat{U}_{\mathrm{c}}
\end{array}\right)=\boldsymbol{T}_{\mathrm{lp}}\left(\begin{array}{c}
\hat{p}_{\mathrm{f}} \\
\hat{U}_{\mathrm{f}}
\end{array}\right)
$$

with $\boldsymbol{T}_{\text {lp }}$ given by

$$
\boldsymbol{T}_{\mathrm{lp}}=\boldsymbol{T}_{\mathrm{B}} \boldsymbol{T}_{\mathrm{r}} \boldsymbol{T}_{\mathrm{t}}
$$

Matrix multiplication with (115), a similar relations for $\boldsymbol{T}_{\mathrm{t}}$, and (117) results in

$$
\boldsymbol{T}_{\mathrm{lp}}=\left(\begin{array}{cc}
c_{\mathrm{B}} c_{\mathrm{t}}-\frac{T_{\mathrm{a}}}{T_{\mathrm{t}}} s_{\mathrm{B}} s_{\mathrm{t}}+\frac{i A}{c \rho_{0}} c_{\mathrm{B}} R_{\mathrm{r}} s_{\mathrm{t}} & c_{\mathrm{B}} R_{\mathrm{r}} c_{\mathrm{t}}+i \frac{c \rho_{0}}{A} c_{\mathrm{B}} s_{\mathrm{t}}+i \frac{T_{\mathrm{a}}}{T_{\mathrm{t}}} \frac{c \rho_{0}}{A} s_{\mathrm{B}} c_{\mathrm{t}} \\
\frac{i A}{c \rho_{0}} s_{\mathrm{B}} c_{\mathrm{t}}-\frac{A^{2}}{c^{2} \rho_{0}^{2}} R_{\mathrm{r}} s_{\mathrm{B}} s_{\mathrm{t}}+\frac{i A}{c \rho_{0}} \frac{T_{\mathrm{a}}}{T_{\mathrm{t}}} c_{\mathrm{B}} s_{\mathrm{t}} & \frac{T_{\mathrm{a}}}{T_{\mathrm{t}}} c_{\mathrm{B}} c_{\mathrm{t}}-s_{\mathrm{B}} s_{\mathrm{t}}+\frac{i A}{c \rho_{0}} R_{\mathrm{r}} s_{\mathrm{B}} c_{\mathrm{t}}
\end{array}\right) .
$$

Furthermore

$$
\operatorname{det} \boldsymbol{T}_{\mathrm{lp}}=\operatorname{det} \boldsymbol{T}_{\mathrm{B}} \operatorname{det} \boldsymbol{T}_{\mathrm{r}} \operatorname{det} \boldsymbol{T}_{\mathrm{t}}=\frac{T_{\mathrm{a}}}{T_{\mathrm{t}}} .
$$

At the intersection point

$$
\hat{p}_{\mathrm{b}}=\hat{p}_{\mathrm{c}}=\hat{p}_{\mathrm{f}} \equiv \hat{p}
$$

gives, with (118),

$$
\hat{p}=T_{11 \mathrm{lp}} \hat{p}+T_{12 \mathrm{lp}} \hat{U}_{\mathrm{f}}
$$




$$
\hat{U}_{\mathrm{c}}=T_{211 \mathrm{p}} \hat{p}+T_{221 \mathrm{p}} \hat{U}_{\mathrm{f}} .
$$

So

$$
\hat{p}=\frac{T_{121 \mathrm{p}}}{1-T_{11 \mathrm{lp}}} \hat{U}_{\mathrm{f}}
$$

and, with (121),

$$
\hat{U}_{\mathrm{c}}=\frac{-\frac{T_{\mathrm{a}}}{T_{\mathrm{t}}}+T_{22 \mathrm{lp}}}{1-T_{11 \mathrm{p}}} \hat{U}_{\mathrm{f}} .
$$

For the resonator tube

$$
\begin{aligned}
& \hat{p}_{\mathrm{b}}=c_{\mathrm{R}} \hat{p}_{\mathrm{a}}+\mathrm{i} \frac{c \rho_{0}}{A} s_{\mathrm{R}} \hat{U}_{\mathrm{a}}, \\
& \hat{U}_{\mathrm{b}}=\mathrm{i} \frac{A}{c \rho_{0}} s_{\mathrm{R}} \hat{p}_{\mathrm{a}}+c_{\mathrm{R}} \hat{U}_{\mathrm{a}} .
\end{aligned}
$$

For an open resonator tube, as discussed here,

$$
\hat{p}_{\mathrm{a}}=0 \text {. }
$$

With (127), (128), and (125) this gives

$$
\hat{U}_{\mathrm{b}}=\frac{c_{\mathrm{R}}}{\mathrm{i} c \rho_{0} s_{\mathrm{R}}} \frac{T_{121 \mathrm{p}}}{1-T_{11 \mathrm{p}}} \hat{U}_{\mathrm{f}}
$$

Mass conservation at the intersection gives

$$
\hat{U}_{\mathrm{f}}=\hat{U}_{\mathrm{b}}+\hat{U}_{\mathrm{c}} .
$$

With (126) and (130) we see that nonzero solutions can be found only if

$$
0=-1+T_{11 \mathrm{lp}}+\frac{A c_{\mathrm{R}}}{\mathrm{i} c \rho_{0} S_{\mathrm{R}}} T_{12 \mathrm{lp}}-\frac{T_{\mathrm{a}}}{T_{\mathrm{t}}}+T_{22 \mathrm{lp}} .
$$

Substituting the matrix elements from (120) gives

$$
\begin{aligned}
0= & c_{\mathrm{B}} c_{\mathrm{t}}-s_{\mathrm{B}} s_{\mathrm{t}}+c_{\mathrm{B}} \frac{c_{\mathrm{R}}}{s_{\mathrm{R}}} s_{\mathrm{t}}-1+\frac{T_{\mathrm{a}}}{T_{\mathrm{t}}}\left(c_{\mathrm{B}} c_{\mathrm{t}}-s_{\mathrm{B}} s_{\mathrm{t}}+c_{\mathrm{R}} \frac{s_{\mathrm{B}}}{s_{\mathrm{R}}} c_{\mathrm{t}}-1\right) \\
& +\frac{\mathrm{i} A R_{\mathrm{r}}}{c \rho_{0}}\left(c_{\mathrm{B}} s_{\mathrm{t}}+s_{\mathrm{B}} c_{\mathrm{t}}-c_{\mathrm{B}} \frac{c_{\mathrm{R}}}{s_{\mathrm{R}}} c_{\mathrm{t}}\right) .
\end{aligned}
$$

This relation leads to rather simple expressions for the oscillation frequency and the onset temperature as follows: from the real part we get

$$
\frac{T_{\mathrm{a}}}{T_{\mathrm{t}}}=\frac{c_{\mathrm{B}} c_{\mathrm{t}}-s_{\mathrm{B}} s_{\mathrm{t}}+\frac{c_{\mathrm{R}}}{s_{\mathrm{R}}} c_{\mathrm{B}} s_{\mathrm{t}}-1}{1-c_{\mathrm{B}} c_{\mathrm{t}}+s_{\mathrm{B}} s_{\mathrm{t}}-\frac{c_{\mathrm{R}}}{s_{\mathrm{R}}} s_{\mathrm{B}} c_{\mathrm{t}}}
$$


and from the imaginary part

$$
\frac{s_{\mathrm{t}}}{c_{\mathrm{t}}}+\frac{s_{\mathrm{B}}}{c_{\mathrm{B}}}=\frac{c_{\mathrm{R}}}{s_{\mathrm{R}}} .
$$

So the oscillation frequency is given by

$$
\tan \left(k L_{\mathrm{R}}\right)\left[\tan \left(k L_{\mathrm{t}}\right)+\tan \left(k L_{\mathrm{B}}\right)\right]=1 .
$$

Substitution of (135) in (134) results in

$$
\frac{T_{\mathrm{a}}}{T_{\mathrm{t}}}=\frac{c_{\mathrm{B}}}{c_{\mathrm{t}}} .
$$

In other words the onset temperature is given by

$$
T_{\mathrm{t}}=T_{\mathrm{a}} \frac{\cos \left(k L_{\mathrm{t}}\right)}{\cos \left(k L_{\mathrm{B}}\right)} .
$$

Equation (138) shows how $T_{\mathrm{t}}$ depends on the position of the regenerator in the loop. For the system of [38] with $L_{\mathrm{t}}=24 \mathrm{~cm}, L_{\mathrm{B}}=93 \mathrm{~cm}$, and $L_{\mathrm{R}}=2 \mathrm{~m}$ (136) gives $v=82 \mathrm{~Hz}$ and (138) gives and onset temperature of $334 \mathrm{~K}$. In practice the onset temperature will be higher due to dissipative effects, which have been neglected in this treatment.

\subsubsection{Standing-Wave Systems}

In this section we will derive expressions for the onset temperature and the oscillation frequency of the prime mover represented in Fig. 28a. Figure 30 represents the thermoacoustic prime mover, divided in three subsystems. The transfer matrix $\boldsymbol{T}_{\text {ad }}$, relating the pressure variations and the volume flows at the ends, a and $\mathrm{d}$, is equal to the product of the transfer matrices of the three subsystems

$$
\boldsymbol{T}_{\mathrm{ad}}=\boldsymbol{T}_{\mathrm{ab}} \boldsymbol{T}_{\mathrm{bc}} \boldsymbol{T}_{\mathrm{cd}}
$$

Here $\boldsymbol{T}_{\mathrm{ab}}, \boldsymbol{T}_{\mathrm{bc}}$, and $\boldsymbol{T}_{\mathrm{cd}}$ are the transfer matrices for the back side (system ab), of the stack (system bc), and of the resonator tube (system cd) respectively. In particular

$$
\hat{U}_{\mathrm{a}}=T_{21 \mathrm{ad}} \hat{p}_{\mathrm{d}}+T_{22 \mathrm{ad}} \hat{U}_{\mathrm{d}} .
$$

Fig. 30 The thermoacoustic prime mover, divided in three subsystems: ab, bc, and cd

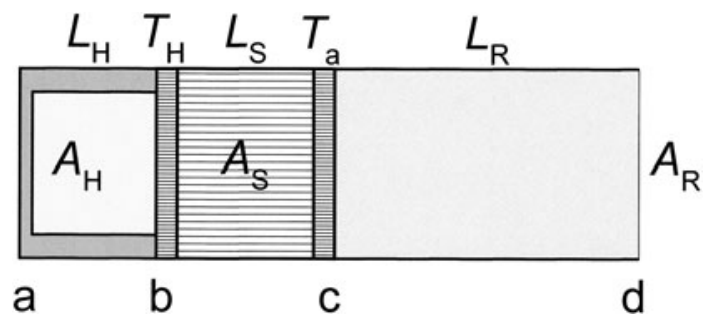


The volume flow at the left and the pressure variation at the tube exit are zero, so $\hat{U}_{\mathrm{a}}=0$ and $\hat{p}_{\mathrm{d}}=0$. Nonzero oscillations are possible only if

$$
T_{22 \mathrm{ad}}=0 .
$$

With (139) Equation (141) gives

$$
0=T_{21 \mathrm{ab}}\left(T_{11 \mathrm{bc}} T_{12 \mathrm{~cd}}+T_{12 \mathrm{bc}} T_{22 \mathrm{~cd}}\right)+T_{22 \mathrm{ab}}\left(T_{21 \mathrm{bc}} T_{12 \mathrm{~cd}}+T_{22 \mathrm{bc}} T_{22 \mathrm{~cd}}\right) .
$$

The transfer matrices $\boldsymbol{T}_{\mathrm{ab}}$ and $\boldsymbol{T}_{\mathrm{cd}}$ are given by (225), and $\boldsymbol{T}_{\mathrm{bc}}$ by (200). Note that $T_{\mathrm{a}}$ and $T_{\mathrm{b}}$ (the temperatures at position a and b) of (200) in this case have to be identified with $T_{\mathrm{H}}$ (the hot-end temperature) and $T_{\mathrm{a}}$ (ambient temperature) respectively, so that here $\Delta \tau=\left(T_{\mathrm{a}}-T_{\mathrm{H}}\right) / T_{\mathrm{a}}$. For simplicity it is assumed that the density and the speed of sound at the hot side are equal to the values at ambient temperature. Substituting the expressions for the matrix elements in (142), using (100) and (103), results in

$$
\begin{aligned}
0= & \tan \left(k L_{\mathrm{H}}\right)\left(\frac{A_{\mathrm{H}}}{A_{\mathrm{R}}} \tan \left(k L_{\mathrm{R}}\right)+\frac{A_{\mathrm{H}}}{A_{\mathrm{S}}} \frac{k L_{\mathrm{S}}}{1-f_{v}}\right) \\
& +\left[1+(\gamma-1) f_{\kappa}\right] \frac{A_{\mathrm{S}}}{A_{\mathrm{R}}} k L_{\mathrm{S}} \tan \left(k L_{\mathrm{R}}\right)-\left(1+g_{\mathrm{S}} \frac{T_{\mathrm{H}}-T_{\mathrm{a}}}{T_{\mathrm{a}}}\right) .
\end{aligned}
$$

Here $L_{\mathrm{H}}, A_{\mathrm{H}}$ are the length and area of the hot side, $L_{\mathrm{S}}, A_{\mathrm{S}}$ are the length and the free-flow area of the stack, and $L_{\mathrm{R}}, A_{\mathrm{R}}$ are the length and area of the resonator. The equations for the real and imaginary parts of (143) result in expressions for $\omega$ and the onset temperature. They depend only on the ratios of the areas. Equation (143) is quite complicated but, with algebraic software, it is quite easy to evaluate. A typical result is given in Fig. 31 where the frequency $v=\omega / 2 \pi$ and the onset temperature are given as functions of the relative half plate distance

$$
r=\frac{y_{0}}{\delta_{\kappa}}
$$

Equation (143) also has solutions with higher harmonics at higher onset temperatures. These are not shown in Fig. 31. The pressure amplitude is determined by the heat input at the hot end.

Fig. 31 Onset temperature and oscillation frequency as functions of the relative plate distance $r$. In this particular case nitrogen is used as the working fluid and $A_{\mathrm{H}}=A_{\mathrm{S}}=A_{\mathrm{R}}$, $L_{\mathrm{H}}=L_{\mathrm{S}}=5 \mathrm{~cm}, L_{\mathrm{R}}=20 \mathrm{~cm}$, $p_{0}=1$ bar, $T_{\mathrm{a}}=300 \mathrm{~K}$

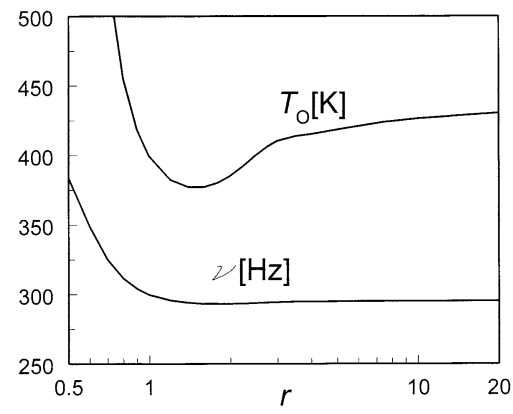




\subsubsection{The Thermoacoustic Cooler}

An example of a thermoacoustic cooler is represented in Fig. 28b. The cooling results from heat shuttling by the gas parcels moving back and forth in the stack as long as the temperature gradient in the stack is smaller than the critical temperature gradient. The enthalpy flow in the stack is essentially nonzero. For the cooling power $\dot{Q}_{\mathrm{L}}$ and the power $P$, applied to the speaker, Equation (20) holds. The power $P$ flows from the speaker to the left heat exchanger, transmitted by the sound wave. So the system operates due to a traveling wave at the left of the stack. Although these systems are often called standing-wave systems, a traveling waves play an essential role in the operation of the cooler. The enthalpy and entropy flows in the stack are negative (so from the cold to the warm side). This type of cooler is studied extensively by Tijani [37]. In particular he has shown that the COP can be improved by using gas mixtures.

\section{JT Refrigerators}

So far we have treated coolers which use oscillating flows and a regenerator or a stack. In the remaining part of this paper we will treat two important coolers which use a steady flow of the working fluid and counterflow heat exchangers to produce cooling. The first is the Joule-Thomson cooler.

\subsection{System Description}

The Joule-Thomson (JT) cooler is invented by Carl von Linde and William Hampson so this cooler is also called the Linde-Hampson cooler. Basically it is a very simple type of cooler which is widely applied as the (final stage) of liquefaction machines and cryocoolers. It can easily be miniaturized, but it is also used on a very large scale in the liquefaction of natural gas. A schematic diagram of a JT liquefier, is given in Fig. 32. Basically it consists of a compressor, a counterflow heat exchanger, a JT valve, and a reservoir. In this discussion we assume that the heat exchanger is ideal. This means that it has no flow resistance and that the gas at the low-pressure side leaves the heat exchanger with room temperature. In Fig. 32 the pressures and temperatures refer to the case of a nitrogen liquefier. At the inlet of the compressor the gas is at room temperature $(300 \mathrm{~K})$ and a pressure of 1 bar (point a). After compression it is at $300 \mathrm{~K}$ and a pressure of 200 bar (point b). Next it enters the heat exchanger where it is precooled. It leaves the exchanger at point c. After the JT expansion, at point $\mathrm{d}$, it has a temperature of $77.36 \mathrm{~K}$ and a pressure of 1 bar. The liquid fraction is $x$. The liquid leaves the system at the bottom of the reservoir (point e) and the remaining gas flows into the counterflow heat exchanger at the cold side (point $f$ ). As said before, it leaves the heat exchanger with a temperature which is equal to room temperature (point a). 
Fig. 32 Schematic diagram of a JT liquefier. A fraction $x$ of the compressed gas is removed as liquid. At room temperature it is supplied as gas at $1 \mathrm{bar}$, so that the system is in the steady state. The symbols a-f refer to points in the Ts-diagram of Fig. 33

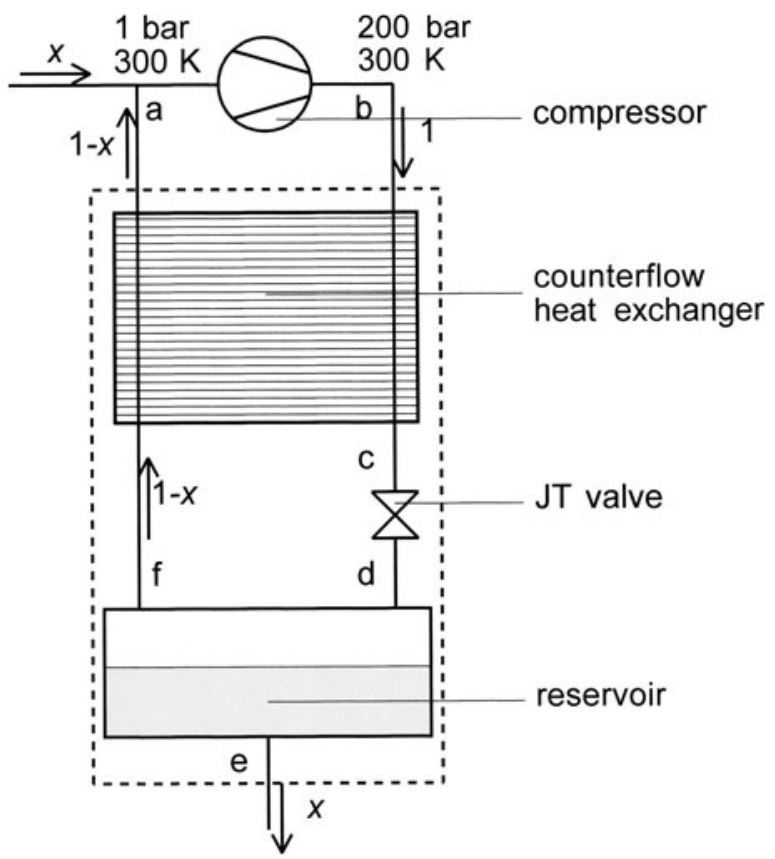

\subsection{Thermodynamic Analysis}

Now we will calculate the liquid fraction $x$. For this we need the thermodynamic properties of nitrogen which can be obtained from the Ts-diagram in Fig. 33 [43]. The calculation of $x$ becomes surprisingly simple if we consider the system indicated by the dotted rectangle in Fig. 32. This is an adiabatic system (no heat exchange with its surroundings) with rigid walls and it is in the steady state. For such a system the first law (see (1)) reduces to a conservation law for the enthalpy. This reads as follows

$$
h_{\mathrm{b}}=x h_{\mathrm{e}}+(1-x) h_{\mathrm{a}}
$$

so

$$
x=\frac{h_{\mathrm{a}}-h_{\mathrm{b}}}{h_{\mathrm{a}}-h_{\mathrm{e}}} .
$$

The labels a, b, c, d, e, and f correspond with the points in Figs. 32 and 33. Clearly there can only be liquefaction if $x>0$. As $h_{\mathrm{a}}>h_{\mathrm{e}}$ this means

$$
h_{\mathrm{a}}>h_{\mathrm{b}} .
$$

This is true for nitrogen at room temperature. For every substance there is a certain temperature $T_{\mathrm{i}}$, the so-called inversion temperature, where $(\partial h / \partial p)_{\mathrm{T}}$ changes sign. For a starting temperature below the inversion temperature liquefaction is possible via a JT expansion.

With the enthalpy values, obtained from Fig. 33, we get with (146) $x=0.07$. Now the value of the enthalpy in point $\mathrm{d}$ can be calculated with $h_{\mathrm{d}}=x h_{\mathrm{e}}+(1-x) h_{\mathrm{f}}=$ 


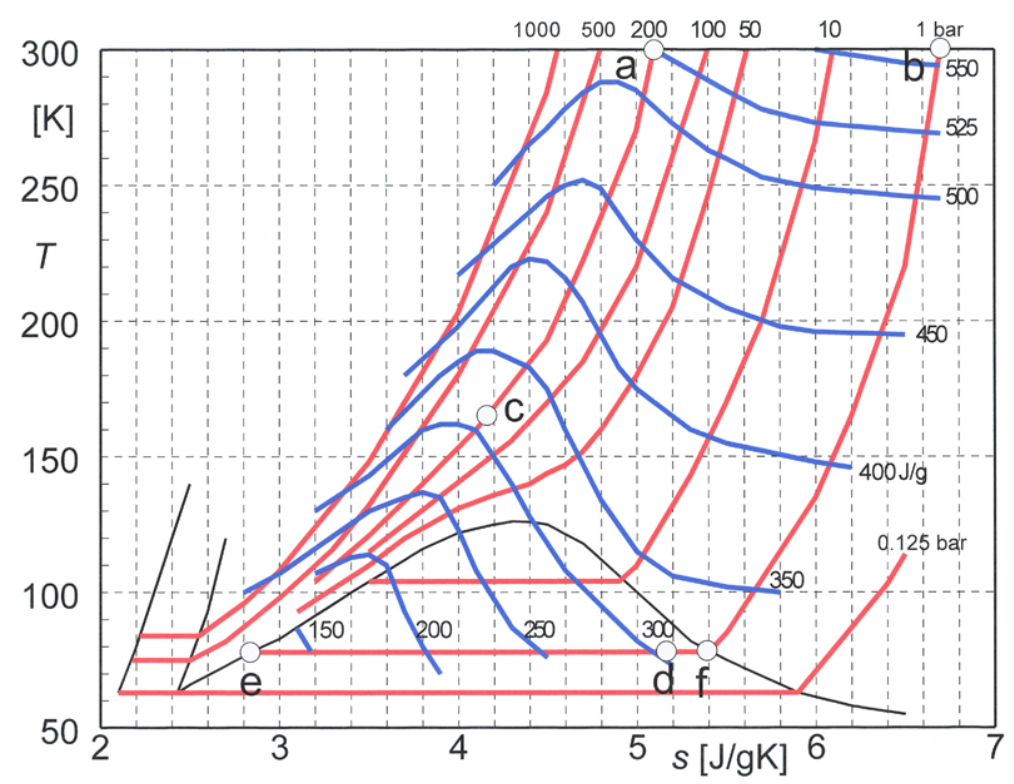

Fig. 33 (Color online) $T s$-diagram of nitrogen with isobars, isenthalps, and the lines of coexistence. The pressures are given in bar, the specific enthalpy in $\mathrm{J} / \mathrm{g}$. The dots, labeled a-f, correspond with the points in Fig. 32

$307 \mathrm{~J} / \mathrm{g}$. Similarly $s_{\mathrm{d}}=x s_{\mathrm{e}}+(1-x) s_{\mathrm{f}}=5.2 \mathrm{~J} / \mathrm{g} \mathrm{K}$. The enthalpy of point $\mathrm{d}$ is equal to the enthalpy in point c. Following the isenthalp of $307 \mathrm{~J} / \mathrm{g}$ (which runs "parallel" with the isenthalp of $300 \mathrm{~J} / \mathrm{g}$ ) we find the starting temperature of the expansion at point $\mathrm{c}$. It is about $T_{\mathrm{c}}=165 \mathrm{~K}$. The corresponding entropy can be read from the diagram $s_{\mathrm{c}}=4.2 \mathrm{~J} / \mathrm{g} \mathrm{K}$.

There are several irreversible processes in this cooler. The entropy per gram, produced at the JT expansion, is

$$
s_{\mathrm{iJT}}=s_{\mathrm{d}}-s_{\mathrm{c}}=5.2-4.2=1.0 \mathrm{~J} / \mathrm{g} \mathrm{K} .
$$

The total entropy production, per gram, is

$$
s_{\text {itot }}=x s_{\mathrm{e}}+(1-x) s_{\mathrm{a}}-s_{\mathrm{b}}=1.33 \mathrm{~J} / \mathrm{g} \mathrm{K} .
$$

The difference between $s_{\mathrm{itot}}$ and $s_{\mathrm{iJT}}$ is due to the irreversible processes in the heat exchanger. On the low-temperature side of the heat exchanger the high-pressure gas leaves the heat exchanger with temperature $T_{\mathrm{c}}=165 \mathrm{~K}$ while the temperature of the gas entering the heat exchanger is $T_{\mathrm{e}}=77.36 \mathrm{~K}$. This difference in temperatures between the two sides is fundamental and cannot be avoided even in the case of an ideal heat exchanger.

If the JT-cooler is used as a cooler (and not as a liquefier) irreversible processes can be reduced by using gas mixtures instead of pure fluids. Also the high pressures, which are 200 bar in the case of pure nitrogen, can be reduced significantly [44]. 


\section{Dilution Refrigerators}

Dilution refrigerators are fantastic machines: starting from $4.2 \mathrm{~K}$ they provide continuous cooling to temperatures as low as $2 \mathrm{mK}$ without moving parts in the lowtemperature region. Also from the thermodynamic and hydrodynamic point of view they are interesting since they are based on a unique combination of a Fermi liquid $\left({ }^{3} \mathrm{He}\right)$ and a superfluid $\left({ }^{4} \mathrm{He}\right)$. The ${ }^{4} \mathrm{He}$ component is at rest, but it is superfluid so it allows easy flow of ${ }^{3} \mathrm{He}$ through the ${ }^{4} \mathrm{He}$ (up to a certain critical velocity). The flow of ${ }^{3} \mathrm{He}$ in the dilute side is driven by a pressure gradient, balancing the viscous forces, just like any other normal fluid. In the superfluid ${ }^{4} \mathrm{He}$, which is at rest, the pressure gradient is balanced by an osmotic pressure gradient. The latter is possible thanks to the fact that, at $T=0$, a mixture of ${ }^{3} \mathrm{He}$ and ${ }^{4} \mathrm{He}$ separates in one phase, which is pure ${ }^{3} \mathrm{He}$ (the concentrated phase), and another phase (the dilute phase) which is not pure ${ }^{4} \mathrm{He}$ but contains only $93.4 \%{ }^{4} \mathrm{He}$ and the rest $(6.6 \%)$ is ${ }^{3} \mathrm{He}$. Due to the Fermi character of the ${ }^{3} \mathrm{He}$ the concentration of $6.6 \%$ results in an osmotic pressure (which is a property of the superfluid ${ }^{4} \mathrm{He}$ ) of $\Pi_{0}=2209 \mathrm{~Pa}$ even at absolute zero. This allows a pressure drop, to a maximum of $\Pi_{0}$, for driving the ${ }^{3} \mathrm{He}$ through the dilute channel.

In this section the essence of the operation of dilution refrigerators will be given from a thermodynamical point of view. For details we refer to Refs. [45, 46] and the references therein.

\subsection{Main Components}

Figure 34 is a schematic diagram of the dilution unit of a dilution refrigerator. The working fluid is ${ }^{3} \mathrm{He}$ which is circulated by pumps at room temperature. These pumps (not shown in Fig. 34) bring the pressure of the ${ }^{3} \mathrm{He}$ to a value $p_{\mathrm{c}}$ which usually is a few hundred millibar. The ${ }^{3} \mathrm{He}$ enters the cryostat and is precooled by a helium bath

Fig. 34 (Color online) Schematic diagram of the low-temperature part of a dilution refrigerator (the dilution unit). The components are described in the text

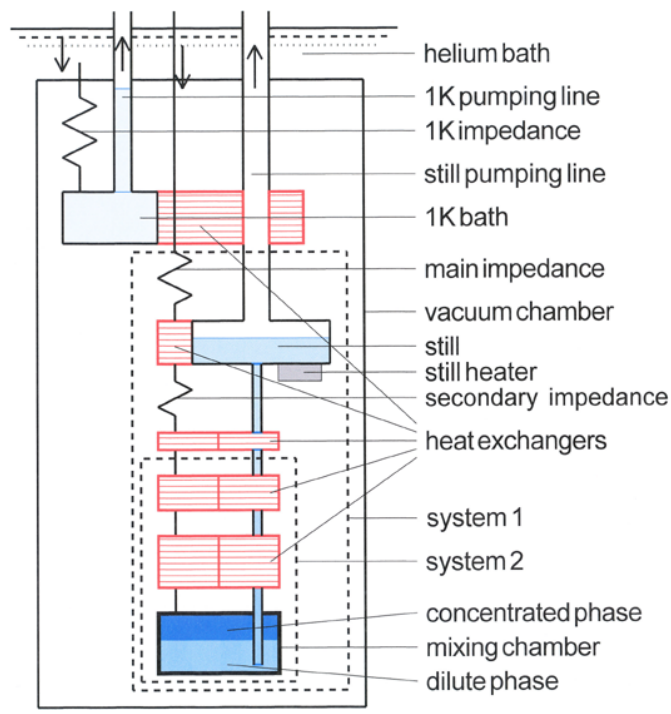


at $4.2 \mathrm{~K}$. Next, the ${ }^{3} \mathrm{He}$ enters the vacuum chamber where it is further cooled down to a temperature of $1.2-1.5 \mathrm{~K}$ by the so-called $1 \mathrm{~K}$ bath, which is a pumped ${ }^{4} \mathrm{He}$ bath. The pressure of the incoming ${ }^{3} \mathrm{He}, p_{\mathrm{c}}$, is larger than the ${ }^{3} \mathrm{He}$ vapor pressure $p_{\mathrm{v}}$ at the temperature of the $1 \mathrm{~K}$ bath, so the ${ }^{3} \mathrm{He}$ condenses (goes to the liquid phase). The heat of condensation is removed by the $1 \mathrm{~K}$ bath. Next the ${ }^{3} \mathrm{He}$ enters the main impedance. This is a capillary with a very large flow resistance that ensures that $p_{\mathrm{c}}$ is large enough to guarantee condensation on the $1 \mathrm{~K}$ bath for the design ${ }^{3} \mathrm{He}$ flow rate ${ }^{*}{ }_{3}$. Next the ${ }^{3} \mathrm{He}$ exchanges heat with the still (to be explained later) which is at a temperature $T_{\mathrm{S}}$ of around 500 to $700 \mathrm{mK}$. At $700 \mathrm{mK}$ the ${ }^{3} \mathrm{He}$ vapor pressure is about $100 \mathrm{~Pa}$. This is a low pressure, but yet large enough that there is a risk that the liquid ${ }^{3} \mathrm{He}$ starts to boil again. So, the ${ }^{3} \mathrm{He}$ enters a secondary impedance which has a flow resistance so that the pressure in the still heat exchanger is larger than the vapor pressure at $T_{\mathrm{S}}$. Next the ${ }^{3} \mathrm{He}$ enters a set of counterflow heat exchangers flowing down where it is cooled by a cold flow of ${ }^{3} \mathrm{He}$ in the other side which flows up. Usually the heat exchangers in the high-temperature range of above $50 \mathrm{mK}$ are of the tube-in-tube type. In the colder regions they are more complicated since they need a large surface area to reduce the Kapitza resistance. After leaving the coldest heat exchanger the ${ }^{3} \mathrm{He}$ enters the mixing chamber.

In the mixing chamber the ${ }^{3} \mathrm{He}$ passes the so-called phase boundary. The phase boundary separates the concentrated phase (practically $100 \%{ }^{3} \mathrm{He}$ ) and the dilute phase $\left(6.6 \%{ }^{3} \mathrm{He}\right.$ and $93.6 \%$ superfluid $\left.{ }^{4} \mathrm{He}\right)$ which are in equilibrium. In passing the phase boundary the ${ }^{3} \mathrm{He}$ is diluted. The heat, needed for the dilution, will be calculated later on and is the cooling power of the refrigerator. The ${ }^{3} \mathrm{He}$ leaves the mixing chamber in the dilute phase. On its way up the cold ${ }^{3} \mathrm{He}$ in the dilute phase cools the warm flow of ${ }^{3} \mathrm{He}$ in the concentrated phase flowing down through the heat exchangers until it enters the still. On its way up the ${ }^{3} \mathrm{He}$ concentration is gradually reduced from $6.6 \%$ in the mixing chamber to only $0.7 \%$ in the still. Yet the vapor in the still is practically $(96 \%)$ pure ${ }^{3} \mathrm{He}$. A heating power $\dot{Q}_{\mathrm{S}}$ (to be calculated below) is supplied to the still to maintain a steady flow of ${ }^{3} \mathrm{He}$ through the system. The pressure in the still is reduced by pumps at room temperature to pressures of about $10 \mathrm{~Pa}$ and pressurized again to a pressure $p_{\mathrm{c}}$ so the cycle is closed.

In the next sections the basic thermodynamics of the dilution unit will discussed. For a more detailed treatment the reader is referred to Ref. [46].

\subsection{The $1 \mathrm{~K}$ Bath}

We can understand the function of the $1 \mathrm{~K}$ bath and the still surprisingly well from the thermodynamic properties of pure ${ }^{3} \mathrm{He}$. Therefore we give here the $H_{\mathrm{m}}-T$ diagram of ${ }^{3} \mathrm{He}$ (Fig. 35) [47]. In this section we will first show why a precooling stage, such as the $1 \mathrm{~K}$ bath, is necessary. In order to do this we look at what would happen if it would not be there. Consider the system inside the dashed contour indicated as system 1 in Fig. 34. The essence of this system is that only pure ${ }^{3} \mathrm{He}$ passes the system boundaries. In the steady state the first law reads

$$
0=\dot{Q}+\stackrel{*}{n}_{3}\left(H_{\mathrm{m} 1}-H_{\mathrm{m} 2}\right)
$$


Fig. 35 (Color online) $H_{\mathrm{m}}-T$ diagram of ${ }^{3} \mathrm{He}$. Lines of constant pressure are given together with the phase diagram. The blue dots refer to points discussed in the text

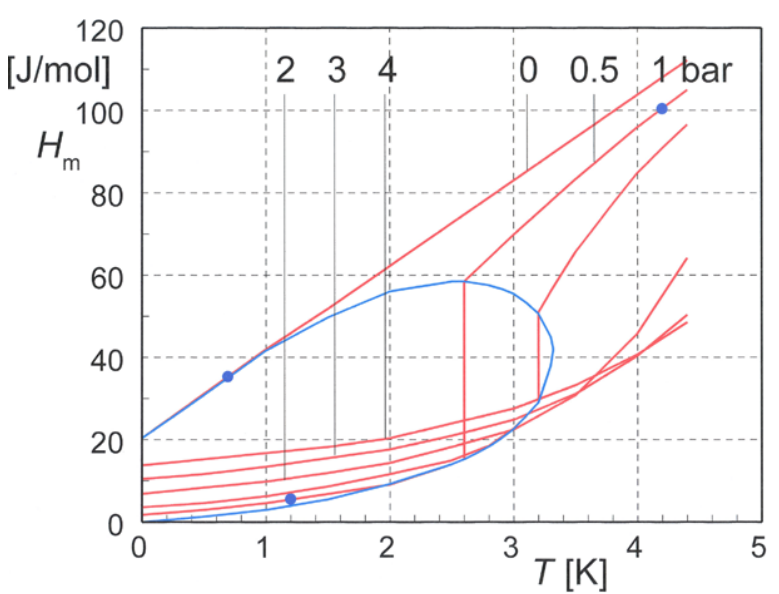

where the index 1 (2) applies to the entrance (exit) of the ${ }^{3} \mathrm{He}$ flow at a molar flow rate of ${ }^{*}$ and $\dot{Q}$ is a heating power, supplied somewhere to the system. If there would be no $1 \mathrm{~K}$ bath the temperature of the incoming ${ }^{3} \mathrm{He}$ would be $4.2 \mathrm{~K}$. With a pressure of 0.5 bar the molar enthalpy can be obtained from Fig. 35 to be $H_{\mathrm{m} 1}(0.5 \mathrm{bar}, 4.2 \mathrm{~K})=$ $99 \mathrm{~J} / \mathrm{mol}$. If the vapor from the still leaves the system at a temperature of $0.7 \mathrm{~K} H_{\mathrm{m} 2}$ $(0$ bar, $0.7 \mathrm{~K})=35 \mathrm{~J} / \mathrm{mol}$. With these numbers (150) gives

$$
\dot{Q}=\stackrel{*}{n}_{3}(35-99)=-64 \frac{\mathrm{J}}{\mathrm{mol}} \stackrel{*}{n}_{3}
$$

Equation (151) shows that a positive $n_{3}^{*}$ is only possible if the applied heat is negative, in other words: if there is some form of external cooling. This is provided by the $1 \mathrm{~K}$ bath.

Due to the presence of the $1 \mathrm{~K}$ bath ${ }^{3} \mathrm{He}$ enters system 1 as liquid at $1.2 \mathrm{~K}$ instead of as gas at $4.2 \mathrm{~K}$. Now Fig. 35 shows that $H_{\mathrm{m} 1}=6 \mathrm{~J} / \mathrm{mol}$. Substituting this value in (150) gives

$$
\dot{Q}_{\mathrm{S}}=\stackrel{*}{n}_{3}(35-6)=29 \frac{\mathrm{J}}{\mathrm{mol}} \stackrel{*}{n}_{3} .
$$

This heat usually is supplied at the still, hence the label S.

Note that the heating power at the still (see (152)) is calculated without using the mixture properties. The reason is that only pure ${ }^{3} \mathrm{He}$ is passing the boundaries of system 1 . However, if we consider system 2 in Fig. 34 pure ${ }^{3} \mathrm{He}$ enters at the left, but ${ }^{3} \mathrm{He}$ leaves the system in the dilute phase. In this case the enthalpy has to be replaced by the so-called osmotic enthalpy. In this way it can be shown that the temperature at the dilute side is typically half the value of the concentrated side [45]. This illustrates the high cooling power of the dilute phase which can be used e.g. for thermal grounding of electrical wiring and for cooling radiations shields.

Figure 35 shows that the enthalpy of ${ }^{3} \mathrm{He}$ at $4.2 \mathrm{~K}$ can be reduced by increasing the pressure from 0.5 bar to 2 to 3 bar. At $4.2 \mathrm{~K}$ the minimum $H_{\mathrm{m}} \approx 42 \mathrm{~J} / \mathrm{mol}$, which is just above the enthalpy of the vapor at $0.7 \mathrm{~K}(35 \mathrm{~J} / \mathrm{mol})$. Hence it is possible to reach 
Fig. 36 Schematic drawing of a dry dilution refrigerator. In the left corner is the two-stage PTR cooling the radiation shields and the incoming ${ }^{3} \mathrm{He}$ (also via the regenerator of the PTR)

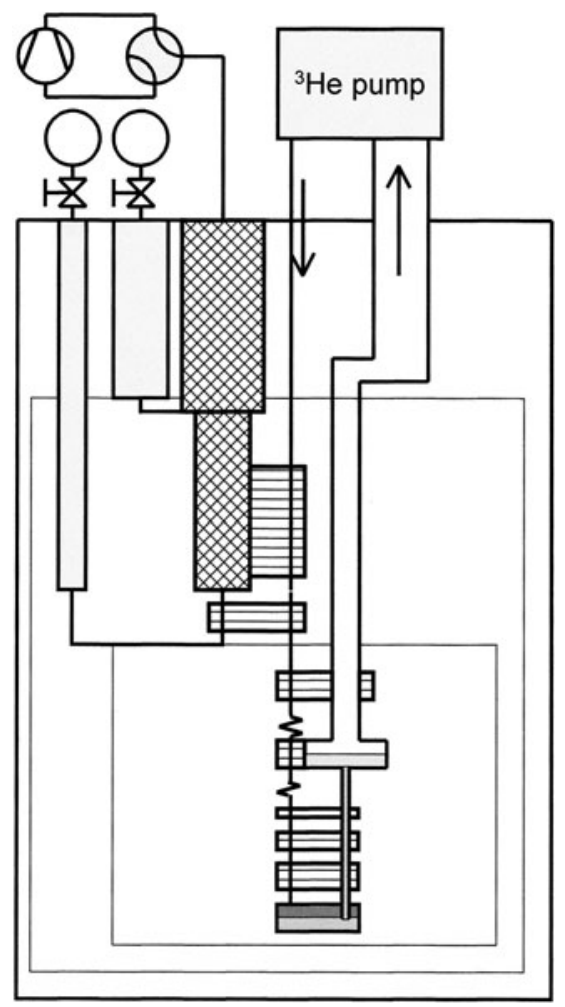

a steady state, with a nonzero ${ }^{3} \mathrm{He}$ circulation, if the incoming pressure is a few bar and a fairly simple heat exchanger is used between the incoming and outgoing ${ }^{3} \mathrm{He}$ $[47,48]$.

The so-called dry systems, shown in Fig. 36, use a cryocooler instead of liquid helium and liquid nitrogen to precool the ${ }^{3} \mathrm{He}$ and cool the radiation shields. The precooling temperature of the ${ }^{3} \mathrm{He}$ is around $3 \mathrm{~K}$ [49]. In that case the enthalpy of the incoming ${ }^{3} \mathrm{He}$ is already low enough and hardly any heat exchanger between vapor and gas is needed at the warm end of the dilution unit.

\subsection{The Mixing Chamber}

In this Subsection the origin of the cooling power of the mixing chamber will be explained. The left part of Fig. 37 represents the mixing chamber. The dark-gray area is the concentrated phase and the light-gray area the dilute phase. The right part represents the temperature profile. The ${ }^{3} \mathrm{He}$ enters the mixing chamber with temperature $T_{\mathrm{i}}$ and the temperature of the phase boundary, which separates the concentrated and the dilute phase, is $T_{\mathrm{M}}$. On its way from the inlet (at $l=L_{\mathrm{c}}$ ) to the phase boundary (at $l=0$ ) the temperature of the ${ }^{3} \mathrm{He}$ is reduced from $T_{\mathrm{i}}$ to $T_{\mathrm{M}}$. In this section we will neglect viscous heating. In the concentrated phase the total energy flow

$$
E=\dot{Q}_{\mathrm{c}}(l)+\stackrel{*}{n}_{3} H_{3}
$$


Fig. 37 Left: schematic drawing of the mixing chamber. The dark area is the concentrated phase and the lighter area the dilute phase. The systems $\mathrm{a}$ and $\mathrm{b}$ are explained in the text. The right part represents the temperature profile inside the mixing chamber
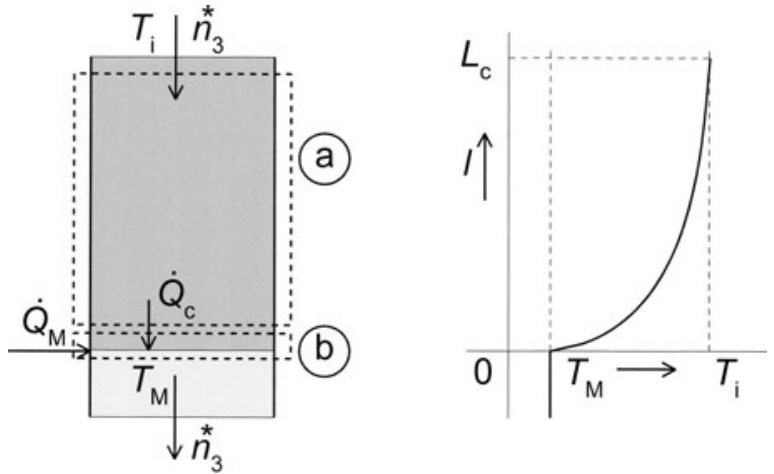

is constant as can be derived from applying the first law to system a of Fig. 37. In (153) $\dot{Q}_{\mathrm{c}}$ is the heat flow due to heat conduction and $H_{3}$ is the molar enthalpy of ${ }^{3} \mathrm{He}$ in the concentrated phase (at very low temperatures this is pure ${ }^{3} \mathrm{He}, x_{\mathrm{c}}=1$ ). In a well-designed dilution refrigerator the heat flow at the inlet is practically zero $\dot{Q}_{\mathrm{c}}\left(L_{\mathrm{c}}\right) \approx 0$. In that case the heat flow to the phase boundary $(l=0$, system a in Fig. 37) is given by

$$
\dot{Q}_{\mathrm{c}}(0)=\stackrel{*}{n}_{3}\left[H_{3}\left(T_{\mathrm{i}}\right)-H_{3}\left(T_{\mathrm{M}}\right)\right] .
$$

The total heat flow $\dot{Q}_{\mathrm{t}}$ to the phase boundary is the sum of $\dot{Q}_{\mathrm{c}}(0)$ and the externally-applied heating power $\dot{Q}_{\mathrm{M}}$

$$
\dot{Q}_{\mathrm{t}}=\dot{Q}_{\mathrm{c}}(0)+\dot{Q}_{\mathrm{M}}
$$

In order to express $\dot{Q}_{\mathrm{t}}$ in terms of the thermodynamic ${ }^{3} \mathrm{He}$ properties we consider system b. Its upper border is just above the phase boundary and the lower system border just below the phase boundary. The mixing is reversible so $\dot{Q}_{\mathrm{t}}$ can be derived from the second law of thermodynamics, applied to system b, with $\dot{S}_{\mathrm{i}}=0$

$$
0=\frac{\dot{Q}_{\mathrm{t}}}{T_{\mathrm{M}}}+\stackrel{*}{n}_{3}\left(S_{3}-S_{\mathrm{d}}\right) .
$$

In (156) $S_{3}\left(T_{\mathrm{M}}\right)$ is the molar entropy of pure ${ }^{3} \mathrm{He}$ and $S_{\mathrm{d}}\left(T_{\mathrm{M}}, x_{\mathrm{s}}\right)$ is the entropy of one mol ${ }^{3} \mathrm{He}$ in the dilute phase, and $x_{\mathrm{s}}$ is the saturated ${ }^{3} \mathrm{He}$ concentration of the dilute phase (which is also a function of $T$ ).

In the concentrated and the dilute phase the ${ }^{3} \mathrm{He}$ behaves as an ideal Fermi gas with the molar entropy given by

$$
S_{\mathrm{F}}=\frac{\pi^{2}}{2} R \frac{T}{T_{\mathrm{F}}(x)}
$$

with $T_{\mathrm{F}}$ the Fermi temperature. For the concentrated phase

$$
S_{3}(T)=\frac{\pi^{2}}{2} R \frac{T}{T_{\mathrm{F}}(1)}
$$


where $T_{\mathrm{F}}(1)$ is the Fermi temperature of pure ${ }^{3} \mathrm{He}$. For the entropy of the dilute phase we can write

$$
S_{\mathrm{d}}\left(T, x_{\mathrm{s}}\right)=S_{\mathrm{F}}\left(T, x_{\mathrm{s}}\right)=\frac{\pi^{2}}{2} R \frac{T}{T_{\mathrm{F}}\left(x_{\mathrm{s}}\right)}
$$

where $T_{\mathrm{F}}\left(x_{\mathrm{s}}\right)$ is the Fermi temperature of the dilute phase. So with (156)

$$
\dot{Q}_{\mathrm{t}}=\stackrel{*}{n}_{3} \frac{\pi^{2}}{2} R T_{\mathrm{M}}^{2}\left(\frac{1}{T_{\mathrm{F}}\left(x_{\mathrm{s}}\right)}-\frac{1}{T_{\mathrm{F}}(1)}\right) .
$$

Equation (160) shows that the cooling power of the mixing chamber is due to the difference in the Fermi temperatures of the dilute and the concentrated phase. Now the Fermi temperature is given by

$$
T_{\mathrm{F}}=\frac{1}{8}\left(\frac{3}{\pi}\right)^{2} \frac{h^{2}}{m_{3}^{*} k_{\mathrm{B}}}\left[\frac{N_{\mathrm{A}}}{V_{\mathrm{m}}(x)}\right]^{2 / 3}
$$

with $k_{\mathrm{B}}$ Boltzmann's constant, $V_{\mathrm{m}}$ the volume (of the mixture) containing one mol of ${ }^{3} \mathrm{He}$, and $m_{3}^{*}$ an effective mass. In the dilute phase $m_{3}^{*}=2.46 m_{3}$ and in the concentrated phase $m_{3}^{*}=2.8 m_{3}$, with $m_{3}$ the mass of the bare ${ }^{3} \mathrm{He}$ atom. The factors 2.46 and 2.8 are about the same, so the main difference between $T_{\mathrm{F}}\left(x_{\mathrm{s}}\right)$ and $T_{\mathrm{F}}(1)$ is due to the difference in molar volume. At low temperatures $V_{\mathrm{m}}\left(x_{\mathrm{s}}\right)=426 \mathrm{~cm}^{3}$ and $V_{\mathrm{m}}(1)=37 \mathrm{~cm}^{3}$, leading to $T_{\mathrm{F}}\left(x_{\mathrm{s}}\right)=393 \mathrm{mK}$ and $T_{\mathrm{F}}(1)=1.8 \mathrm{~K}$ respectively. So the cooling power of a dilution refrigerator is really based on the increase of molar volume (dilution) of the ${ }^{3} \mathrm{He}$ !

\section{Conclusion}

The combination of the first and second law of thermodynamics for open, inhomogeneous, systems with the concepts enthalpy flow, entropy flow, and entropy production, provides a powerful tool for analyzing a large variety of thermal machines such as cryocoolers and heat engines. A deep understanding of the system operation can be obtained, usually with simple mathematics, which leads to elegant expressions with general validity.

Acknowledgements M.E.H. van Dongen and M.E.H. Tijani are acknowledged for their stimulating interest and carefully reading the manuscript. The staff and students of the Institute of Refrigeration and Cryogenics of the Zhejiang University in Hangzhou (China) are acknowledged for their stimulating interest.

Open Access This article is distributed under the terms of the Creative Commons Attribution Noncommercial License which permits any noncommercial use, distribution, and reproduction in any medium, provided the original author(s) and source are credited. 


\section{Appendix A: Some Useful Formulae}

\section{A.1 General Relations}

The molar entropy satisfies

$$
\mathrm{d} S_{\mathrm{m}}=\frac{C_{\mathrm{p}}}{T} \mathrm{~d} T-\left(\frac{\partial V_{\mathrm{m}}}{\partial T}\right)_{\mathrm{p}} \mathrm{d} p .
$$

The molar enthalpy

$$
\mathrm{d} H_{\mathrm{m}}=T \mathrm{~d} S_{\mathrm{m}}+V_{\mathrm{m}} \mathrm{d} p
$$

and also

$$
\mathrm{d} H_{\mathrm{m}}=C_{\mathrm{p}} \mathrm{d} T+H_{\mathrm{p}} \mathrm{d} p
$$

with $C_{\mathrm{p}}$ the molar heat capacity at constant pressure and

$$
H_{\mathrm{p}}=\left(\frac{\partial H_{\mathrm{m}}}{\partial p}\right)_{\mathrm{T}}=V_{\mathrm{m}}-\left(\frac{\partial V_{\mathrm{m}}}{\partial T}\right)_{\mathrm{p}} T,
$$

where $V_{\mathrm{m}}$ the molar volume. We can also write

$$
H_{\mathrm{p}}=V_{\mathrm{m}}\left(1-T \alpha_{\mathrm{V}}\right)
$$

with the volumetric expansion coefficient

$$
\alpha_{\mathrm{V}}=\frac{1}{V_{\mathrm{m}}}\left(\frac{\partial V_{\mathrm{m}}}{\partial T}\right)_{\mathrm{p}} .
$$

\section{A.2 Ideal Gas Relations}

In most cryocoolers helium is used as the working fluid. At temperatures above $20 \mathrm{~K}$ and pressures below 30 bar helium behaves as an ideal gas. In that case

$$
p V_{\mathrm{m}}=R T
$$

In (168) $R$ is the molar ideal gas constant. ${ }^{4}$ The relation for the entropy change becomes

$$
\mathrm{d} S_{\mathrm{m}}=C_{\mathrm{p}} \frac{\mathrm{d} T}{T}-R \frac{\mathrm{d} p}{p}
$$

Other useful expression are

$$
\mathrm{d} S_{\mathrm{m}}=C_{\mathrm{V}} \frac{\mathrm{d} T}{T}+R \frac{\mathrm{d} V_{\mathrm{m}}}{V_{\mathrm{m}}}
$$

\footnotetext{
${ }^{4}$ Here $R=8.314510 \mathrm{~J} / \mathrm{mol} \mathrm{K}$ is the molar ideal gas constant, not to be confused with the specific ideal gas constant which depends on the molar mass of the gas and is expressed in $J / \mathrm{kg} \mathrm{K}$.
} 
and

$$
\begin{aligned}
\mathrm{d} S_{\mathrm{m}} & =C_{\mathrm{p}} \frac{\mathrm{d} V_{\mathrm{m}}}{V_{\mathrm{m}}}+C_{\mathrm{V}} \frac{\mathrm{d} p}{p}, \\
\gamma & =\frac{C_{\mathrm{p}}}{C_{\mathrm{V}}} .
\end{aligned}
$$

An analytical expression for $S_{\mathrm{m}}$ of an ideal gas is

$$
S_{\mathrm{m}}(p, T)=C_{\mathrm{p}} \ln \frac{T}{T_{0}}-R \ln \frac{p}{p_{0}} .
$$

For the enthalpy

$$
\mathrm{d} H_{\mathrm{m}}=C_{\mathrm{p}} \mathrm{d} T \text {. }
$$

As $C_{\mathrm{p}}$ is constant for an ideal gas we may write

$$
H_{\mathrm{m}}(p, T)=C_{\mathrm{p}} T .
$$

Similarly we have for the molar internal energy

$$
U_{\mathrm{m}}(p, T)=C_{\mathrm{V}} T \text {. }
$$

\section{Appendix B: The Volume-Flow Equation}

In this appendix the so-called volume-flow equation is derived. The internal energy of the gas in a volume $V$ can be expressed as

$$
U=\int_{V} \frac{U_{\mathrm{m}}}{V_{\mathrm{m}}} \mathrm{d} V
$$

If the pressure is homogeneous and the gas is ideal then $U_{\mathrm{m}}=C_{\mathrm{V}} T$ and $V_{\mathrm{m}}=R T / p$ so, (177) gives

$$
U=\frac{C_{\mathrm{V}}}{R} p V .
$$

Note that (178) holds even though the temperature in $V$ is not homogeneous. Since $H_{\mathrm{m}}=C_{\mathrm{p}} T$ the enthalpy flows into the control volume are given by $\stackrel{*}{H}{ }_{k}=\stackrel{*}{n_{k}} C_{\mathrm{p}} T_{k}$. With $\stackrel{*}{n}_{k}=p \stackrel{*}{V}_{k} / R T_{k}$ we get

$$
\stackrel{*}{H}_{k}=\frac{C_{\mathrm{p}}}{R} p \stackrel{*}{V_{k}}
$$

The system is adiabatic so $\sum \dot{Q}_{k}=0$ and also $P=0$, so (1), with (178) and (179), reads

$$
\frac{C_{\mathrm{V}}}{R} V \frac{\mathrm{d} p}{\mathrm{~d} t}+\frac{C_{\mathrm{V}}}{R} p \frac{\mathrm{d} V}{\mathrm{~d} t}=\frac{C_{\mathrm{p}}}{R} p \sum \stackrel{*}{V}_{k}-p \sum \frac{\mathrm{d} V_{k}}{\mathrm{~d} t}
$$


With

$$
\frac{\mathrm{d} V}{\mathrm{~d} t}=\sum \frac{\mathrm{d} V_{k}}{\mathrm{~d} t}
$$

and $C_{\mathrm{V}}+R=C_{\mathrm{p}}$ and $\gamma=C_{\mathrm{p}} / C_{\mathrm{V}}$ we get our final result

$$
\frac{V}{\gamma p} \frac{\mathrm{d} p}{\mathrm{~d} t}=\sum \stackrel{*}{V}_{k}-\sum \frac{\mathrm{d} V_{k}}{\mathrm{~d} t} .
$$

\section{Appendix C: The Harmonic Model}

In many thermal machines the variation of the system parameters, such as pressure, temperature, and flow, is periodic with angular frequency $\omega$. For small amplitudes, the variations can be well approximated in the linear regime. In these cases the variations can be described in terms of $\sin \omega t$ and $\cos \omega t$ which is called the harmonic approximation. The harmonic approximation treats systems in the steady state as the amplitudes are considered time independent. Before discussing the harmonic model in more detail we have to pay attention to some delicate aspects of temperature variations and the volume flow (or velocity).

Consider the temperature $T$ of the gas, just left of the heat exchanger $\mathrm{X}_{3}$ of the PTR in Fig. 16. As long as gas flows to the left $(\stackrel{*}{n}<0)$ the temperature at this point is constant and equal to $T_{\mathrm{a}}$. However, if the gas flows to the right, back into $\mathrm{X}_{3}(\stackrel{*}{n}>0)$, the temperature is higher (see Fig. 17). The $T-t$ dependence is given by the curve, labeled $T_{\text {real }}$ in Fig. 38. If the molar flow rate at the hot end is given by

$$
\stackrel{*}{n}=n_{\mathrm{A}} \cos \omega t
$$

then the time dependence of the temperature is of the form

$$
T=T_{\mathrm{a}}+T_{\mathrm{A}}(\cos \omega t-|\cos \omega t|) .
$$

This is clearly not harmonic. In the harmonic model a temperature-time dependence is used which is represented by the dotted line, labeled $T_{\text {harm }}$ in Fig. 38, and is of the form

$$
T=T_{\mathrm{a}}+T_{\mathrm{A}} \cos \omega t .
$$

Yet, using (185) instead of (184) gives the correct value for the enthalpy flow in the pulse tube. The reason is that the average enthalpy flow is given by

$$
\bar{H}=C_{\mathrm{p}} \bar{*} \delta T
$$

and that $\overline{|\cos x| \cos x}=0$, so (184) and (185), with (183), give the same result. So the harmonic expression for the temperature (185) may be used even though the actual $T-t$ dependence deviates from the harmonic dependence.

In the steady state the average velocity of all gas particles is zero: i.e. after one cycle they are at the same position as before. However, the volume flow through a 
Fig. 38 The full line, labeled $T_{\text {real }}$, represents the actual temperature-time dependence of the gas in the pulse tube close to the hot heat exchanger. The dotted curve, labeled $T_{\text {harm }}$, gives the temperature dependence as used in the harmonic model. The curve, labeled $\stackrel{*}{n}$, represents the flow

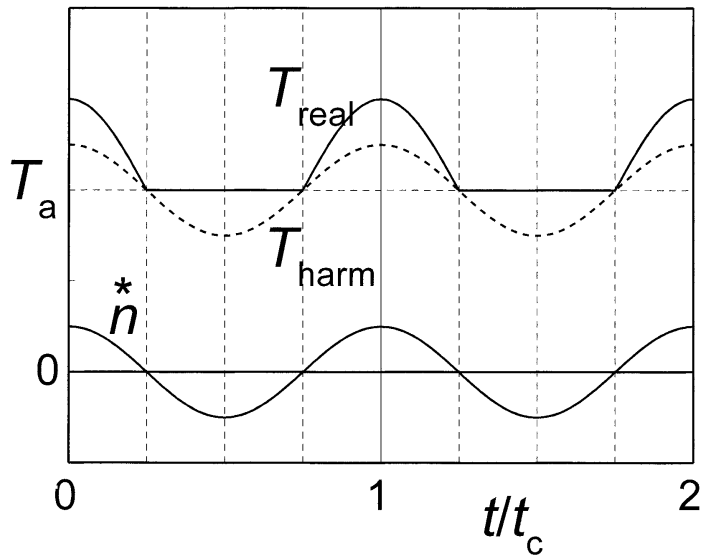

fixed surface, or velocity of the gas particles passing a fixed surface, contains a DC contribution even if the mass (molar) flow through that surface is zero. Starting from $\stackrel{\bar{*}}{n}=\stackrel{*}{V} / V_{\mathrm{m}}=0$ with $V_{\mathrm{m}}=V_{\mathrm{m} 0}+\delta V_{\mathrm{m}}$ gives, for small $\delta V_{\mathrm{m}}$,

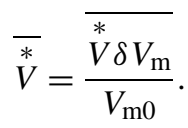

Usually $\stackrel{*}{V}$ and $\delta V_{\mathrm{m}}$ both are of first order (e.g. in the pressure variation), so the average volume flow is of second-order. This means that it plays an important role e.g. in the enthalpy flow since this is also of second-order. With the volume flow given by $\stackrel{*}{V}=v A$, with $v$ the velocity and $A$ the surface area, we see that the average velocity is also nonzero in second order.

Instead of using $\sin \omega t$ and $\cos \omega t$ it is advantages to use the complex formalism since this reduces the number of variables and equations by a factor of two. In the complex formalism of e.g. a pressure variation $\delta p$ the complex amplitude $\hat{p}$ is introduced defined by

$$
\delta p=\operatorname{Re}\left(\hat{p} \mathrm{e}^{\mathrm{i} \omega t}\right) .
$$

The time average of second-order quantities, such as enthalpy flow,

$$
\stackrel{*}{H}=\stackrel{*}{n} \delta H_{\mathrm{m}}
$$

become

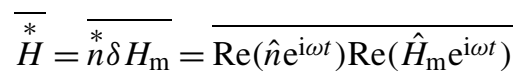

or

$$
\bar{*} \stackrel{*}{H}=\frac{1}{2}\left(\operatorname{Re} \hat{n} \operatorname{Re} \hat{H}_{\mathrm{m}}+\operatorname{Im} \hat{n} \operatorname{Im} \hat{H}_{\mathrm{m}}\right)=\frac{1}{2} \operatorname{Re}\left(\hat{n} \hat{H}_{\mathrm{m}}^{*}\right)
$$

where $\hat{H}_{\mathrm{m}}^{*}$ is the complex conjugate of $\hat{H}_{\mathrm{m}}$. 
The complex formalism allows the use of transfer functions which relate the pressure variation $\hat{p}_{\mathrm{a}}$ and volume flow $\hat{U}_{\mathrm{a}}$ at position a with $\hat{p}_{\mathrm{b}}$ and $\hat{U}_{\mathrm{b}}$ at position $\mathrm{b}$ of a certain control volume via the linear relations

$$
\begin{aligned}
& \hat{p}_{\mathrm{a}}=T_{11} \hat{p}_{\mathrm{b}}+T_{12} \hat{U}_{\mathrm{b}}, \\
& \hat{U}_{\mathrm{a}}=T_{21} \hat{p}_{\mathrm{b}}+T_{22} \hat{U}_{\mathrm{b}} .
\end{aligned}
$$

The transfer relations (192) and (193) can also be written in terms of the transfer matrix

$$
\boldsymbol{T}=\left(\begin{array}{ll}
T_{11} & T_{12} \\
T_{21} & T_{22}
\end{array}\right)
$$

so that

$$
\left(\begin{array}{c}
\hat{p} \\
\hat{U}
\end{array}\right)_{\mathrm{a}}=\boldsymbol{T}\left(\begin{array}{c}
\hat{p} \\
\hat{U}
\end{array}\right)_{\mathrm{b}} .
$$

The transfer-matrix elements have different dimensions. The $T_{11}$ and $T_{22}$ are dimensionless, while $T_{12}$ is in $\mathrm{Pa} \mathrm{s} / \mathrm{m}^{3}$ and $T_{21}$ in $\mathrm{m}^{3} / \mathrm{Pa} \mathrm{s}^{5}$

Components is series can be expressed in products of transfer matrices. A useful property is that the determinant of the product matrix is equal to the product of the determinants of each of the individual matrices. Below expressions will be given for some important components of thermal machines. The derivations are beyond the scope of this paper. The expressions can be very complicated, containing unfamiliar functions like Bessel functions and tanh-functions of complex parameters. This makes it hard to grasp quickly the essence of what is going on. But we start with a simple example.

For an adiabatic volume $V$, in which gas flows in at position a and out at position b (Fig. 4a), we get from (33) that the transfer matrix is given by

$$
\boldsymbol{T}_{\mathrm{V}}=\left(\begin{array}{cc}
1 & 0 \\
\mathrm{i} \omega \frac{V}{\gamma p_{0}} & 1
\end{array}\right)
$$

and

$$
\operatorname{det} \boldsymbol{T}_{\mathrm{V}}=1 \text {. }
$$

The transfer matrix for stacks can be derived from expressions in Ref. [35]. The relationships tend to be very complicated. Here we give the relations for an ideal gas as the working fluid, and stack plates with very large heat capacity. The differential equations for the pressure and volume flow variations are as follows

$$
\frac{\mathrm{d} \hat{p}}{\mathrm{~d} x}=-\frac{\mathrm{i} \omega \rho_{0}}{1-f_{v}} \frac{1}{A_{\mathrm{S}}} \hat{U},
$$

\footnotetext{
${ }^{5}$ In some important cases simpler relations between the dynamic properties at the two ends of the system can be obtained if not the pressure and volume flow are related, but the pressure and molar flux (or mass flux).
} 


$$
\frac{\mathrm{d} \hat{U}}{\mathrm{~d} x}=-\left[1+(\gamma-1) f_{\kappa}\right] \frac{\mathrm{i} \omega A_{\mathrm{S}}}{\gamma p_{0}} \hat{p}+\frac{\left(f_{\kappa}-f_{\nu}\right)}{\left(1-f_{\nu}\right)\left(1-P_{\mathrm{r}}\right) T_{0}} \frac{\mathrm{d} T_{0}}{\mathrm{~d} x} \hat{U} .
$$

For a short stack of length $L_{\mathrm{S}}$ and small temperature difference these correspond with a transfer matrix

$$
\boldsymbol{T}_{\mathrm{S}}=\left(\begin{array}{cc}
1 & \frac{\mathrm{i} \omega \rho_{0}}{1-f_{v}} \frac{L_{\mathrm{S}}}{A_{\mathrm{S}}} \\
{\left[1+(\gamma-1) f_{\kappa}\right] \mathrm{i} \omega \frac{V_{\mathrm{S}}}{\gamma p_{0}}} & 1-g_{\mathrm{S}} \frac{T_{\mathrm{b}}-T_{\mathrm{a}}}{T_{\mathrm{b}}}
\end{array}\right) .
$$

Here $A_{\mathrm{S}}$ is the free-flow surface area of the stack and

$$
V_{\mathrm{S}}=L_{\mathrm{S}} A_{\mathrm{S}}
$$

The function $g_{\mathrm{S}}$ is defined by

$$
g_{\mathrm{S}}=\frac{f_{\kappa}-f_{v}}{\left(1-f_{v}\right)\left(1-P_{\mathrm{r}}\right)},
$$

where $f_{\kappa}$ and $f_{v}$ are the so-called Rott functions given by

$$
f_{\kappa, v}=\frac{\tanh z_{\kappa, v}}{z_{\kappa, v}} \quad \text { with } z_{\kappa, v}=(1+\mathrm{i}) \frac{y_{0}}{\delta_{\kappa, v}}
$$

with $2 y_{0}$ the plate distance. The functions $f(z)$ are even functions of $z$ so they are functions of

$$
z^{2}=\mathrm{i} \frac{2 y_{0}^{2}}{\delta^{2}}
$$

Due to (25) and (26) the Rott functions are not functions of $\sqrt{\omega}$ but of $\omega$. For $y_{0} / \delta \ll 1$ Equation (200) reduces to

$$
\boldsymbol{T}_{\mathrm{S}}=\left(\begin{array}{cc}
1 & 3 \frac{\eta}{y_{0}^{2}} \frac{L_{\mathrm{S}}}{A_{\mathrm{S}}} \\
i \omega \frac{V_{\mathrm{S}}}{p_{0}} & \frac{T_{\mathrm{a}}}{T_{\mathrm{b}}}
\end{array}\right)
$$

This is the same as for a slit with Poiseuille flow, isothermal compression, and zero porosity. For $y_{0} / \delta \gg 1(200)$ becomes

$$
\boldsymbol{T}_{\mathrm{S}}=\left(\begin{array}{cc}
1 & \mathrm{i} \omega \rho_{0} \frac{L_{\mathrm{S}}}{A_{\mathrm{S}}} \\
\mathrm{i} \omega \frac{V_{\mathrm{S}}}{\gamma p_{0}} & 1
\end{array}\right)
$$

This is the same as from a sound duct for $L \rightarrow 0$ (see (225)).

Equation (200) holds for small temperature differences. For large temperature differences, assuming a linear temperature profile, and taking effective values for the Rott functions, (198) and (199) can be integrated. This results in expressions containing Bessel functions, similar to the expressions for the regenerator, which we will describe now. 
For a very simple regenerator with flow conductance $R_{\mathrm{r}}$ and zero porosity the transfer matrix is

$$
\boldsymbol{T}_{\mathrm{r}}=\left(\begin{array}{cc}
1 & R_{\mathrm{r}} \\
0 & T_{\mathrm{a}} / T_{\mathrm{b}}
\end{array}\right)
$$

and

$$
\operatorname{det} \boldsymbol{T}_{\mathrm{r}}=\frac{T_{\mathrm{a}}}{T_{\mathrm{b}}} .
$$

For a regenerator with nonzero flow resistance but with constant specific flow resistance $z_{\mathrm{r}}$, defined by

$$
\frac{\mathrm{d} p}{\mathrm{~d} l}=-\eta z_{\mathrm{r}} v
$$

with the viscosity $\eta$ given by

$$
\eta=\eta_{\mathrm{a}} \sqrt{\frac{T}{T_{\mathrm{a}}}}
$$

(with $\eta_{\mathrm{a}}$ the viscosity at the temperature $T_{\mathrm{a}}$ ), nonzero porosity, an ideal gas, and a linear temperature profile with slope $\mathrm{d} T_{0} / \mathrm{d} x$, the derivation is given in Ref. [25]. The result is

$$
\boldsymbol{T}_{\mathrm{r}}=\left(\begin{array}{ll}
T_{11} & T_{12} \\
T_{21} & T_{22}
\end{array}\right)_{\mathrm{r}}
$$

with elements

$$
\begin{aligned}
& T_{11 r}=F_{2}\left(u_{\mathrm{b}}\right) F_{3}\left(u_{\mathrm{a}}\right)+F_{1}\left(u_{\mathrm{b}}\right) F_{4}\left(u_{\mathrm{a}}\right), \\
& T_{12 r}=\left[-F_{4}\left(u_{\mathrm{b}}\right) F_{3}\left(u_{\mathrm{a}}\right)+F_{3}\left(u_{\mathrm{b}}\right) F_{4}\left(u_{\mathrm{a}}\right)\right] \frac{1}{k_{\mathrm{r}} T_{\mathrm{b}}}, \\
& T_{21 r}=\left[-F_{1}\left(u_{\mathrm{a}}\right) F_{2}\left(u_{\mathrm{b}}\right)+F_{1}\left(u_{\mathrm{b}}\right) F_{2}\left(u_{\mathrm{a}}\right)\right] k_{\mathrm{r}} T_{\mathrm{a}}, \\
& T_{22 r}=\left[F_{4}\left(u_{\mathrm{b}}\right) F_{1}\left(u_{\mathrm{a}}\right)+F_{3}\left(u_{\mathrm{b}}\right) F_{2}\left(u_{\mathrm{a}}\right)\right] \frac{T_{\mathrm{a}}}{T_{\mathrm{b}}}
\end{aligned}
$$

the parameter $k_{\mathrm{r}}$ given by

$$
k_{\mathrm{r}}=\frac{\mathrm{i} \omega g A_{\mathrm{r}}}{p_{0} \mathrm{~d} T_{0} / \mathrm{d} x}
$$

and the functions $F_{1}$ to $F_{4}$ defined as

$$
\begin{aligned}
& F_{1}(u)=\operatorname{BesselI}(0, u), \\
& F_{2}(u)=\operatorname{BesselK}(0, u), \\
& F_{3}(u)=u \operatorname{BesselI}(1, u), \\
& F_{4}(u)=u \operatorname{BesselK}(1, u) .
\end{aligned}
$$


BesselI and BesselK are the Bessel functions of the first and second kind respectively. The four functions (217)-(220) satisfy

$$
F_{1} F_{4}+F_{2} F_{3}=1 \text {. }
$$

By substitution of (212)-(215) it can be found that, due to (221), also in this case holds

$$
\operatorname{det} \boldsymbol{T}_{\mathrm{r}}=\frac{T_{\mathrm{a}}}{T_{\mathrm{b}}}
$$

The transfer matrix elements are functions of $u_{\mathrm{a}}^{2}$ and $u_{\mathrm{b}}^{2}$ with

$$
u_{\mathrm{a}}^{2}=\frac{16}{25} \frac{\mathrm{i} \omega g \eta_{0} z_{\mathrm{r}}}{\left(\mathrm{d} T_{0} / \mathrm{d} x\right)^{2} p_{0}} T_{\mathrm{a}}^{5 / 2}
$$

and

$$
u_{\mathrm{b}}^{2}=\tau^{5 / 2} u_{\mathrm{a}}^{2}
$$

with

$$
\tau=\frac{T_{\mathrm{b}}}{T_{\mathrm{a}}} .
$$

The transfer matrix for a traveling-wave duct with length $L$ and cross sectional area $A$ with a monochromatic plane wave with wave number $k$ can be derived from (104) and (105). The result is

$$
\boldsymbol{T}_{\mathrm{D}}=\left(\begin{array}{cc}
\cos (k L) & \mathrm{i} \frac{c \rho_{0}}{A} \sin (k L) \\
\mathrm{i} \frac{A}{c \rho_{0}} \sin (k L) & \cos (k L)
\end{array}\right) .
$$

Note that

$$
\operatorname{det} \boldsymbol{T}_{\mathrm{D}}=1 .
$$

For a short $(k L \ll 1)$ tube with volume $V=A L$ Equation (225) reduces to (196).

\section{References}

1. R. Radebaugh, J. Phys., Condens. Matter 21, 164219 (2009)

2. S.R. de Groot, P. Mazur, Non-Equilibrium Thermodynamics (North-Holland, Amsterdam, 1969)

3. T. Kuriyama, R. Hakamada, H. Nakagome, Y. Tokai, M. Sahashi, R. Li, O. Yoshida, K. Matsumoto, T. Hashimoto, Adv. Cryog. Eng. 35B, 1261 (1990)

4. Volumetric Heat Capacities for Regenerator Materials. NIST, Physical and Chemical Properties Division

5. A.T.A.M. de Waele, W. Liang, Cryogenics 48, 417 (2008)

6. H.O. McMahon, W.E. Gifford, Advances in Cryogenic. Engineering 5, 354 (1960)

7. http://www.cryomech.com/

8. J.H. So, G.W. Swift, S. Backhaus, J. Acoust. Soc. Am. 120, 1898 (2006)

9. W.E. Gifford, R.C. Longsworth, Adv. Cryog. Eng. 11, 171 (1966)

10. W.E. Gifford, R.C. Longsworth, Trans. ASME 264 (1964)

11. E.I. Mikulin, A.A. Tarasov, M.P. Shkrebyonock, Adv. Cryog. Eng. 31, 629 (1984) 
12. P. Kittel, Adv. Cryog. Eng. 43, 1927 (1998)

13. J. Zhu, P. Wu, Z. Chen, Cryogenics 30, 514 (1990)

14. S.W. Zhu, S.L. Zhou, N. Yoshimura, Y. Matsubara, Cryocoolers 9, 269 (1997)

15. Y. Matsubara, Proc. 17th Int. Cryogenic Eng. Conf., Inst. Phys. (1998), p. 11

16. S.W. Zhu, Y. Kakimi, K. Fujioka, Y. Matsubara, Cryogenics 37, 461 (1997)

17. Z.H. Gan, W.Q. Dong, L.M. Qiu, X.B. Zhang, H. Sun, Y.L. He, R. Radebaugh, Cryogenics 49, 198 (2009)

18. Y. Matsubara, J.L. Gao, Cryogenics 34, 259 (1994)

19. M.Y. Xu, A.T.A.M. de Waele, Y.L. Ju, Cryogenics 39, 865 (1999)

20. N. Jiang, U. Lindemann, F. Giebeler, G. Thummes, Cryogenics 44, 809 (2004)

21. I.A. Tanaeva, U. Lindemann, N. Jiang, A.T.A.M. de Waele, G. Thummes, Adv. Cryog. Eng. 49B, 1906 (2004)

22. D. Gedeon, Sage: object orientated software for Stirling-type machine design, in Proc. of the 29th Intersociety Energy Conversion and Engineering Conference, vol. 4 (American Institute for Aeronautics and Astronautics, Monterey, 1994), pp. 1902-1907

23. J. Gary, A. O'Gallagher, R. Radebaugh, E. Marquardt, REGEN3.2 Regenerator Model: User Manual. NIST Technical Note (2001)

24. G.W. Swift, Thermoacoustics: A unifying perspective for some engines and refrigerators. The Acoustical Society of America. ISBN 0-7354-0065-2 (2002)

25. A.T.A.M. de Waele, H.W.G. Hooijkaas, P.P. Steijaert, A.A.J. Benschop, Cryogenics 38, 995 (1997)

26. A.T.A.M. de Waele, P.P. Steijaert, J. Gijzen, Cryogenics 37, 313 (1997)

27. V.D. Arp, R.D. McCarty, Thermophysical properties of helium-4 from 0.8 to $1500 \mathrm{~K}$ with pressures to 1500 MPa. NBS Technical Note 1334 (1989)

28. Y. Huang, C. Guobang Chen, V. Arp, J. Chem. Phys. 125, 054505 (2006)

29. R.H. Sherman, F.J. Edeskuty, Ann. Phys. 9, 522 (1960)

30. M.E. Will, A.T.A.M. de Waele, J. Appl. Phys. 98, 044911 (2005)

31. G. Thummes, C. Wang, C. Heiden, Cryogenics 38, 337 (1998)

32. A. Ravex, T. Trollier, J. Tanchon, T. Prouvé, Cryocoolers 14, 157 (2006)

33. R. Radebaugh, E.D. Marquardt, J. Gary, A. O'Gallagher, Cryocoolers 11, 409 (2001)

34. N. Rott, Adv. Appl. Mech. 20, 135 (1980)

35. G.W. Swift, J. Acoust. Soc. Am. 84, 1145 (1988)

36. S.L. Garrett, Resource letter: TA-1. Am. J. Phys. 72, 11 (2004)

37. M.E.H. Tijani, Loudspeaker-driven thermo-acoustic refrigeration. Ph.D. dissertation, Eindhoven University of Technology (2001)

38. S. Backhaus, G.W. Swift, J. Acoust. Soc. Am. 107, 3148 (2000)

39. A.T.A.M. de Waele, J. Sound Vib. 325, 974 (2009)

40. M. Poese, R. Smith, S. Garrett, R. van Gerwen, P. Gosselin, International Institute of Refrigeration, 6th Gustav Lorentzen Conference-Natural Working Fluids, Glasgow UK (2004)

41. K. Tang, T. Lei, T. Jin, G.G. Lin, Z.Z. Xu, Appl. Phys. Lett. 94, 254101 (2009)

42. Y. Li, PhD thesis, Eindhoven University of Technology (2011)

43. NBS Tech Note 648 (1973)

44. G. Venkatarathnam, in Cryogenic Mixed Refrigerant Processes, ed. by K.D. Timmerhaus C. Rizzuto. Int. Cryogenic Monograph Series (Springer, Berlin, 2008)

45. O.V. Lounasmaa, Experimental Principles and Methods Below 1 K (Academic Press, London, 1974)

46. F. Pobell, Matter and Methods at Low Temperatures (Springer, Berlin, 2007)

47. J. Kraus, Cryogenics 17, 173 (1977)

48. A.Th.A.M. de Waele, A.B. Reekers, H.M. Gijsman, Cryogenics 17, 175 (1977)

49. K. Uhlig, Cryogenics 48, 138 (2008) 